\title{
Identification of atrial fibrillation-associated lncRNAs and exploration of their functions based on WGCNA and ceRNA network analyses
}

\author{
Yanhong Liu ${ }^{1, *}$, Xue Liang ${ }^{2, *}$, Jianlong Wang ${ }^{1}$, Yu Wang ${ }^{1}$, Xing Liu², Wenfeng Shangguan², \\ Shuai $\mathrm{Miao}^{2}$, Yingwu Liu ${ }^{1}$ and Zhanquan $\mathrm{Jiao}^{3}$ \\ ${ }^{1}$ Heart Center, Tianjin Third Central Hospital, Tianjin Key Laboratory of Extracorporeal Life Support for Critical Diseases, \\ Artificial Cell Engineering Technology Research Center, Tianjin Institute of Hepatobiliary Disease, Tianjin, China \\ 2 Tianjin Key Laboratory of Ionic-Molecular Function of Cardiovascular disease, Department of Cardiology, Tianjin Institute \\ of Cardiology, The Second Hospital of Tianjin Medical University, Tianjin, China \\ ${ }^{3}$ Department of Cardiology, Tianjin Haihe Hospital, Tianjin Institute of Respiratory Diseases, Tianjin, China
}

\begin{abstract}
Atrial fibrillation (AF) is a common cardiac arrhythmia that induces serious complications. However, pharmacological treatments of AF remain challenging. This study aimed to screen crucial long non-coding RNAs (lncRNAs), microRNAs (miRNAs) and messenger RNA (mRNAs) for AF using the microarray datasets (IncRNAs and mRNAs: GSE79768, GSE115574; miRNAs: GSE68475) collected from the Gene Expression Omnibus database. Weighted correlation network analysis of GSE79768 and GSE115574 datasets identified five modules were highly related to AF status. Among 118 module-related differentially expressed mRNAs, FBXW7, EGFR, CXCR2, ROCK1 and UBE2D1 were considered as hub genes according to the gene significance, module membership and the topological characteristics for the nodes in the protein-protein interaction network. IncRNA MIR100HG and LINC01105 may function by co-expressing with (MIR100HG-ROCK1/FBXW7/UBE2D1, LINC01105-EGFR) mRNAs or sponging miRNAs to regulate mRNAs (LINC01105-miR-125a-3p-EGFR, MIR100HG-miR-200b-3pFBXW7, MIR100HG-miR-561-3p-CXCR2, MIR100HG-miR-548z-UBE2D1). Connectivity Map and Comparative Toxicogenomics Database searches predicted dexamethasone may treat AF by reversing the expression of MIR100HG; artemisinin may reverse the expression of hub DEGs. In conclusion, our results may provide novel molecular mechanisms and potential therapeutic targets and drugs for AF.
\end{abstract}

Key words: Atrial fibrillation - Weighted gene co-expression network analysis - Competitive endogenous RNAs - Small-molecule drugs

\section{Introduction}

Atrial fibrillation (AF) is the most common type of cardiac arrhythmia clinically, with an estimated prevalence rate of $2.31 \%$ in China (Wang et al. 2018) and 5\% in the USA and Europe (Ball et al. 2015). AF not only impairs cardiac functions to induce or exacerbate heart failure (Ruddox et al. 2017), but also causes arterial thromboembolism to increase the risk of developing ischemic stroke (Okumura et al. 2020). These two complications are disabling or deadly,

\footnotetext{
* These authors contributed to this work equally.

Correspondence to: Yingwu Liu, Heart Center, Tianjin Third Central Hospital, Tianjin Key Laboratory of Extracorporeal Life Support for Critical Diseases, Artificial Cell Engineering Technology Research Center, Tianjin Institute of Hepatobiliary Disease, Tianjin 300170 , China

E-mail: yingwuliu_dr@163.com

Zhanquan Jiao, Department of Cardiology, Tianjin Haihe Hospital, Tianjin Institute of Respiratory Diseases, Tianjin,

300350, China

E-mail: jiaozhanquan@126.com
} 
which may impose a tremendous burden on both family and society. However, current pharmacological treatments of AF remain challenging due to low efficacy and high sideeffects. Thus, there is an urgent need to better understand the mechanisms underlying AF to develop more effective and safer therapeutic approaches.

Accumulating evidence indicates that long non-coding RNAs (lncRNAs), a class of non-coding RNA transcripts greater than 200 nucleotides, are involved in the pathogenesis of $\mathrm{AF}$ by directly regulating the transcription of protein-coding messenger RNAs (mRNAs) (Chen et al. 2019a; Lu et al. 2019) or acting as a competing endogenous RNA (ceRNA) to sponge microRNAs (miRNAs) and then indirectly influence the negative regulatory roles of miRNAs on target mRNAs (Wang et al. 2019). Chen et al. found that the expression of lncRNA PCAT-1 was increased in atrial appendages of AF patients compared with those with sinus rhythm (SR). Knockdown of PCAT-1 inhibited the proliferation of cardiac fibroblast cells by reducing its target gene transforming growth factor- $\beta 1$ (TGF- $\beta 1$ ) (Chen et al. 2019a). Lu et al. (2019) observed that the expression of GAS5 was significantly lower in atrial appendages of AF patients than that in SR ones. Upregulation of GAS5 inhibited the growth of AC16 human cardiomyocytes by suppressing the expression of TGF- $\beta 1$ type I receptor (ALK5). Cao et al. (2019) confirmed that PVT1 was increased in atrial muscle tissues from AF patients. Mechanically, PVT1 was reported to act as a sponge for miR-128-3p to facilitate Sp1 expression, which subsequently activated the TGF- $\beta 1 /$ Smad signaling pathway to trigger atrial fibroblast proliferation and collagen production. Du et al. (2020) and Yao et al. (2020) respectively identified the levels of TCONS-00106987 and MIAT were increased in the atria of AF animal models. Downregulation of TCONS-00106987 or MIAT lengthened the atrial effective refractory period (Du et al. 2020; Yao et al. 2020) and attenuated cardiomyocyte apoptosis (Yao et al. 2020). Luciferase reporter assays proved that TCONS-00106987 may promote electrical remodeling via sponging miR-26 to induce the transcription of KCNJ2 (Du et al. 2020); while MIAT regulated miR-133a-3p to induce the high expression of various fibrosis-related genes (i.e. collagen, TGF- $\beta 1$ ) (Yao et al. 2020). Thus, lncRNAs may represent potential therapeutic targets for the blockade of AF. However, AF-associated lncRNAs are still poorly known.

With the developments in microarray or sequencing technology, scholars have attempted to screen crucial lncRNAs for AF from high-throughput data. Ke et al. (2019) found that RP11-99E15.2, RP3-523K23.2, RP11-99E15.2 and RP3-523K23.2 may participate in the pathogenesis of AF by collecting RNA sequencing data in atrial appendages of five cases with $\mathrm{AF}$ and five patients without $\mathrm{AF}$ and predicting the interactions between differentially expressed lncRNAs
(DELs) and genes (DEGs) using the LncADeep software. Wu et al. (2019) identified that ETF1P2 and AP001053.11 may be important $\operatorname{lncRNAs}$ for AF by differential analysis of RNA sequencing data in atrial appendages of six cases with $\mathrm{AF}$ and six controls and construction of a lncRNA-mRNA coexpression network. By integrating three microarray datasets and construction of a ceRNA network, the study of Wu et al. (2020a) revealed that HCG11, KRBOX1-AS1, ACBD5 and RAD52 may compete with WEE1 for hsa-miR-17-5p to influence AF development. Qian et al. (2019) suggested that lncRNA RP11-296014.3 may function in AF by interacting with miR-101 to regulate the TGF- $\beta$ signaling pathway. However, rare studies mined AF-associated lncRNAs by both co-expression and ceRNA network analyses (Wu et al. 2020b). Furthermore, no study associated the lncRNAs with clinical features.

Weighted gene co-expression network analysis (WGCNA) (Langfelder and Horvath 2008) is a powerful systems biology algorithm that clusters highly-correlated genes into the same module and associates modules with clinical phenotypic traits. This method was previously used to screen crucial mRNAs for AF (Tan et al. 2013; Li et al. 2020). In the present study, we aimed to apply WGCNA to identify AF-related co-expression modules that not only included mRNAs, but also included lncRNAs. The mRNAs and lncRNAs in preserved co-expression modules were used for the construction of the IncRNA-miRNA-mRNA ceRNA network. Furthermore, potential small molecular drugs that may have a similar therapeutical function of $\operatorname{lncRNAs}$ or target lncRNAs to treat AF were also predicted. The flow chart used in the present study was presented in Figure 1.

\section{Materials and Methods}

\section{Microarray data}

The Gene Expression Omnibus (GEO, http://www. ncbi.nlm. nih.gov/geo/) database was searched to collect the expression profile data of human AF. Two mRNAs/lncRNAs microarray datasets were obtained, including GSE79768 (Tsai et al. 2016) and GSE115574, both of which were analyzed using the platform of GPL570 ([HG-U133_Plus_2] Affymetrix Human Genome U133 Plus 2.0 Array). GSE79768 contained 14 atrial specimens from seven patients (four females and three males) with AF and 12 from six patients (four females and two males) with SR. One left and one right atrial tissue was collected from each patient, respectively (Tsai et al. 2016). GSE79768 was used for differential analysis and module screening. GSE115574 included 28 atrial tissue samples from 15 patients with $\mathrm{AF}$ ( $n=14$ for left and right atrial tissues, respectively) and 31 atrial tissue samples from 16 patients with SR ( $n=15$ for left atrial tissues, $n=16$ for right atrial 


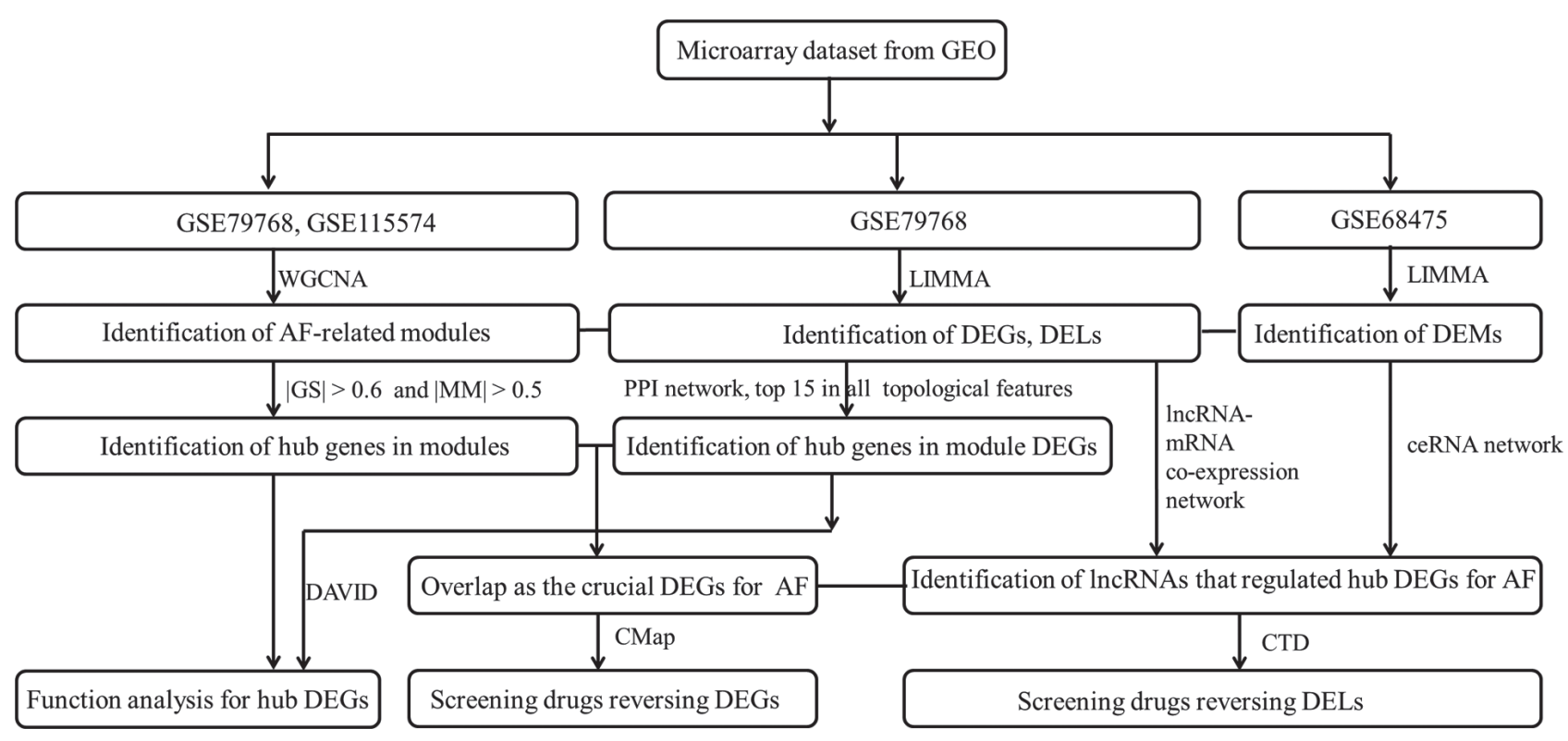

Figure 1. Flowchart used in the present study. GEO, Gene Expression Omnibus; WGCNA, Weighted gene co-expression network analysis; DEGs, differentially expressed genes; DELs, differentially expressed lncRNAs; DEMs, differentially expressed miRNAs; PPI, protein-protein interaction; GS, gene significance; MM, module membership; AF, atrial fibrillation; CMap, connectivity Map; CTD, Comparative Toxicogenomics Database; DAVID, Database for Annotation, Visualization and Integrated Discovery; ceRNA, competing endogenous RNA.

tissues), which was used for module validation. Furthermore, one miRNA microarray dataset (GSE68475) with a relatively large sample size (AF, $n=10$; SR, $n=11$; males) was enrolled (Morishima et al. 2016) for the ceRNA network analysis, which was run in the platform of GPL15018 (Agilent-031181 Unrestricted_Human_miRNA_V16.0_Microarray 030840). The consent of the ethics committee was waived because these data were downloaded from the public database.

\section{Differential expression analysis}

The mRNA and lncRNA gene symbols were re-annotated for the GSE79768 dataset according to the file of Homo_sapiens. GRCh38.95.chr.gtf.gz downloaded from Ensembl human genome database (http://www.ensembl.org/). The Linear Models for Microarray Data (LIMMA) package (version 3.34.7; https://bioconductor.org/packages/release/bioc/html/ limma.html) (Ritchie et al. 2015) for R was used to identify DELs, DEGs in the GSE79768 dataset and differentially expressed miRNAs (DEMs) in the GSE68475 dataset. | $\log _{2} \mathrm{FC}$ (fold change) $\mid>0.5$ and $p$-value $<0.05$ were defined as the statistical threshold. Since no genes in the GSE79768 dataset were found to be significant between whole atrial tissues in patients of AF and SR, the left and right atrial samples were respectively analyzed compared with their controls and then the common DELs and DEGs in left and right atrial samples were selected as the final results. The "pheatmap" package (version: 1.0.8; https://cran.r-project.org/web/packages/ pheatmap) in $\mathrm{R}$ was used to generate the heat map.

\section{WGCNA for identification of important modules associated} with $A F$

The WGCNA package in R (version 1.61; https://cran.rproject.org/web/packages/WGCNA/index.html) (Langfelder and Horvath 2008) was used to identify important co-expression modules and their enriched genes may be candidate targets associated with AF. First, we evaluated the expression and connectivity correlations of all shared RNAs between training (GSE79768) and validation (GSE115574) datasets. Second, the proper soft threshold power $(\beta)$ was chosen based on the scale-free topology criterion. Third, the adjacency matrix between genes in the GSE79768 dataset was calculated, which was converted to a topological overlap matrix (TOM) to construct the cluster dendrogram. The network modules were identified using the DynamicTreeCut algorithm (Langfelder et al. 2008) with a minimum module size of 50 and a minimum cut height of 0.995 . Fourth, the module division was performed for the validation dataset (GSE115574) using the same genes in modules identified from the GSE79768 dataset. The preservation of modules was also confirmed using the modulePreservation statistics, with $\mathrm{Z}$-score $>5$ and $p$-value $<0.05$ defined as the cut-off point (Langfelder et al. 2011). Fifth, the module eigengene (ME) 
that represents the overall expression level of each module was computed. The correlation between ME of each module and clinical features of patients were analyzed with Pearson's correlation and visualized by the heatmap. Significant modules were defined as $p<0.05$. Sixth, to obtain the modules with DELs and DEGs, we mapped the DEGs and DELs into the modules by using the hypergeometric algorithm $[\mathrm{f}(\mathrm{k}$, $\left.\mathrm{N}, \mathrm{M}, \mathrm{n})=\mathrm{C}(\mathrm{k}, \mathrm{M})^{\star} \mathrm{C}(\mathrm{n}-\mathrm{k}, \mathrm{N}-\mathrm{M}) / \mathrm{C}(\mathrm{n}, \mathrm{N})\right]$ (Cao and Zhang 2014). Modules with $p<0.05$ and fold enrichment $>1$ were believed to be especially important. Seventh, to screen hub DEGs in the crucial modules, the gene significance (GS, defined as the correlation between individual genes and clinical variables) and module membership (MM, defined as the correlation between individual genes and module's eigengene) were calculated. The hub genes were identified based on $|\mathrm{GS}|>0.6$ and $|\mathrm{MM}|>0.5$.

\section{Construction of a protein-protein interaction network}

To further screen hub genes from preserved module genes, we attempted to construct a protein-protein interaction (PPI) network. The interactions between module DEGs were predicted by the Search Tool for the Retrieval of Interacting Genes (STRING; version 10.0; http://string db.org/) database (Szklarczyk et al. 2015). PPI pairs with an interaction score $>0.4$ were considered to be significant and chosen to construct the PPI network in the Cytoscape software (version 3.6.1; www.cytoscape.org/). Furthermore, the degree (DC), betweenness (BC), closeness (CC), subgragh (SC) and eigenvector centrality (EC) of each protein in the PPI network was computed using the CytoNCA plugin in the Cytoscape software (http://apps.cytoscape. org/apps/cytonca) (Tang et al. 2015). The proteins ranked

Table 1. Expression of crucial lncRNAs, mRNAs and miRNAs

\begin{tabular}{|c|c|c|c|c|c|c|c|}
\hline \multirow{3}{*}{ RNA type } & \multirow{3}{*}{ Genes } & \multicolumn{6}{|c|}{ Atrial fibrillation $v s$. sinus rhythm } \\
\hline & & \multicolumn{3}{|c|}{ Right atrial appendage } & \multicolumn{3}{|c|}{ Left atrial appendage } \\
\hline & & $\log 2 \mathrm{FC}$ & $p$-value & FDR & $\log 2 \mathrm{FC}$ & $p$-value & FDR \\
\hline \multirow{7}{*}{$\begin{array}{l}\text { lncRNA } \\
\text { (GSE79768) }\end{array}$} & LINC01105 (SILCI) & -0.73 & $4.05 \mathrm{E}-04$ & $9.91 \mathrm{E}-02$ & -0.87 & $8.89 \mathrm{E}-04$ & $2.65 \mathrm{E}-02$ \\
\hline & LINC02502 & 0.53 & $3.31 \mathrm{E}-02$ & $3.45 \mathrm{E}-01$ & 0.88 & $2.75 \mathrm{E}-04$ & $1.77 \mathrm{E}-02$ \\
\hline & TRDN-AS1 & 1.55 & $3.99 \mathrm{E}-05$ & $5.76 \mathrm{E}-02$ & 1.73 & $9.19 \mathrm{E}-05$ & $1.18 \mathrm{E}-02$ \\
\hline & LOC101928304 & 0.70 & $2.88 \mathrm{E}-03$ & $1.78 \mathrm{E}-01$ & 0.69 & $6.72 \mathrm{E}-04$ & $2.36 \mathrm{E}-02$ \\
\hline & MIR100HG & 0.56 & $1.28 \mathrm{E}-02$ & $2.60 \mathrm{E}-01$ & 0.77 & $8.21 \mathrm{E}-04$ & $2.55 \mathrm{E}-02$ \\
\hline & USP3-AS1 & 0.51 & $1.46 \mathrm{E}-02$ & $2.71 \mathrm{E}-01$ & 0.57 & $1.11 \mathrm{E}-02$ & $8.70 \mathrm{E}-02$ \\
\hline & RP11-420K14.2 & 0.85 & $5.79 \mathrm{E}-04$ & $2.23 \mathrm{E}-02$ & 0.81 & $6.41 \mathrm{E}-03$ & $2.08 \mathrm{E}-01$ \\
\hline \multirow{5}{*}{$\begin{array}{l}\text { mRNA } \\
\text { (GSE79768) }\end{array}$} & ROCK1 & 0.52 & $4.35 \mathrm{E}-03$ & $1.89 \mathrm{E}-01$ & 0.78 & $1.20 \mathrm{E}-03$ & $2.91 \mathrm{E}-02$ \\
\hline & EGFR & -0.51 & $5.18 \mathrm{E}-05$ & $5.85 \mathrm{E}-01$ & -0.59 & $6.35 \mathrm{E}-07$ & $4.28 \mathrm{E}-03$ \\
\hline & CXCR2 & 1.01 & $1.76 \mathrm{E}-02$ & $2.83 \mathrm{E}-01$ & 1.50 & $5.54 \mathrm{E}-04$ & $2.18 \mathrm{E}-02$ \\
\hline & FBXW7 & 0.64 & $8.92 \mathrm{E}-04$ & $1.28 \mathrm{E}-01$ & 0.70 & $1.09 \mathrm{E}-04$ & $1.27 \mathrm{E}-02$ \\
\hline & UBE2D1 & 0.75 & $1.59 \mathrm{E}-04$ & $7.49 \mathrm{E}-02$ & 1.27 & $2.06 \mathrm{E}-04$ & $1.60 \mathrm{E}-02$ \\
\hline \multirow{12}{*}{$\begin{array}{l}\text { miRNA } \\
\text { (GSE68475) }\end{array}$} & hsa-miR-125a-3p & 0.54 & $2.79 \mathrm{E}-02$ & $5.16 \mathrm{E}-01$ & & & \\
\hline & hsa-miR-200b & -1.04 & $3.47 \mathrm{E}-03$ & $3.50 \mathrm{E}-01$ & & & \\
\hline & hsa-miR-4314 & 0.80 & $2.38 \mathrm{E}-03$ & $3.50 \mathrm{E}-01$ & & & \\
\hline & hsa-miR-548z & -0.61 & $2.42 \mathrm{E}-03$ & $3.50 \mathrm{E}-01$ & & & \\
\hline & hsa-miR-3926 & 0.60 & $4.44 \mathrm{E}-03$ & $3.50 \mathrm{E}-01$ & & & \\
\hline & hsa-miR-4257 & 0.52 & $5.20 \mathrm{E}-03$ & $3.50 \mathrm{E}-01$ & & & \\
\hline & hsa-miR-3658 & -1.01 & $1.89 \mathrm{E}-02$ & $4.40 \mathrm{E}-01$ & & & \\
\hline & hsa-miR-1183 & 0.68 & $2.57 \mathrm{E}-02$ & $5.00 \mathrm{E}-01$ & & & \\
\hline & hsa-miR-573 & -0.70 & $3.96 \mathrm{E}-02$ & $5.16 \mathrm{E}-01$ & & & \\
\hline & hsa-miR-561 & -0.61 & $4.03 \mathrm{E}-02$ & $5.16 \mathrm{E}-01$ & & & \\
\hline & hsa-miR-323-5p & -0.76 & $4.28 \mathrm{E}-02$ & $5.16 \mathrm{E}-01$ & & & \\
\hline & hsa-miR-3692* & 0.69 & $4.90 \mathrm{E}-02$ & $5.25 \mathrm{E}-01$ & & & \\
\hline
\end{tabular}

FC, fold change; FDR, false discovery rate. 
in the top 15 of all topological characteristics were suggested to be hub genes.

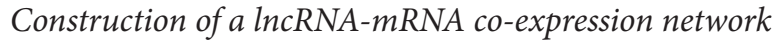

To explain the functions of hub lncRNAs, we attempted to construct a co-expression network between all module DELs and DEGs. WGCNA algorithm was used to calculate their Pearson correlation coefficients (r). The cut-off was set as $r>$ 0.5 and $p<0.01$. The co-expression network was visualized in Cytoscape (version 3.6.1; www.cytoscape.org/).

\section{Construction of a IncRNA-miRNA-mRNA ceRNA network}

miRwalk database (version 2.0; http: //www.zmf.umm.uniheidelberg.de/apps/zmf/mirwalk2) (Dweep and Gretz 2015) is a publicly available resource for the prediction of the interactions between miRNAs and their target genes (including lncRNAs and mRNAs) from multiple existing miRNA-target prediction programs. Thus, the prediction results from miRwalk 2.0 may be more than other individual algorithms. A total of 12 prediction programs (DIANA-microTv4.0, DIANA-microT-CDS, miRanda-rel2010, mirBridge, miRDB4.0, miRmap,

A AF $\quad S R$
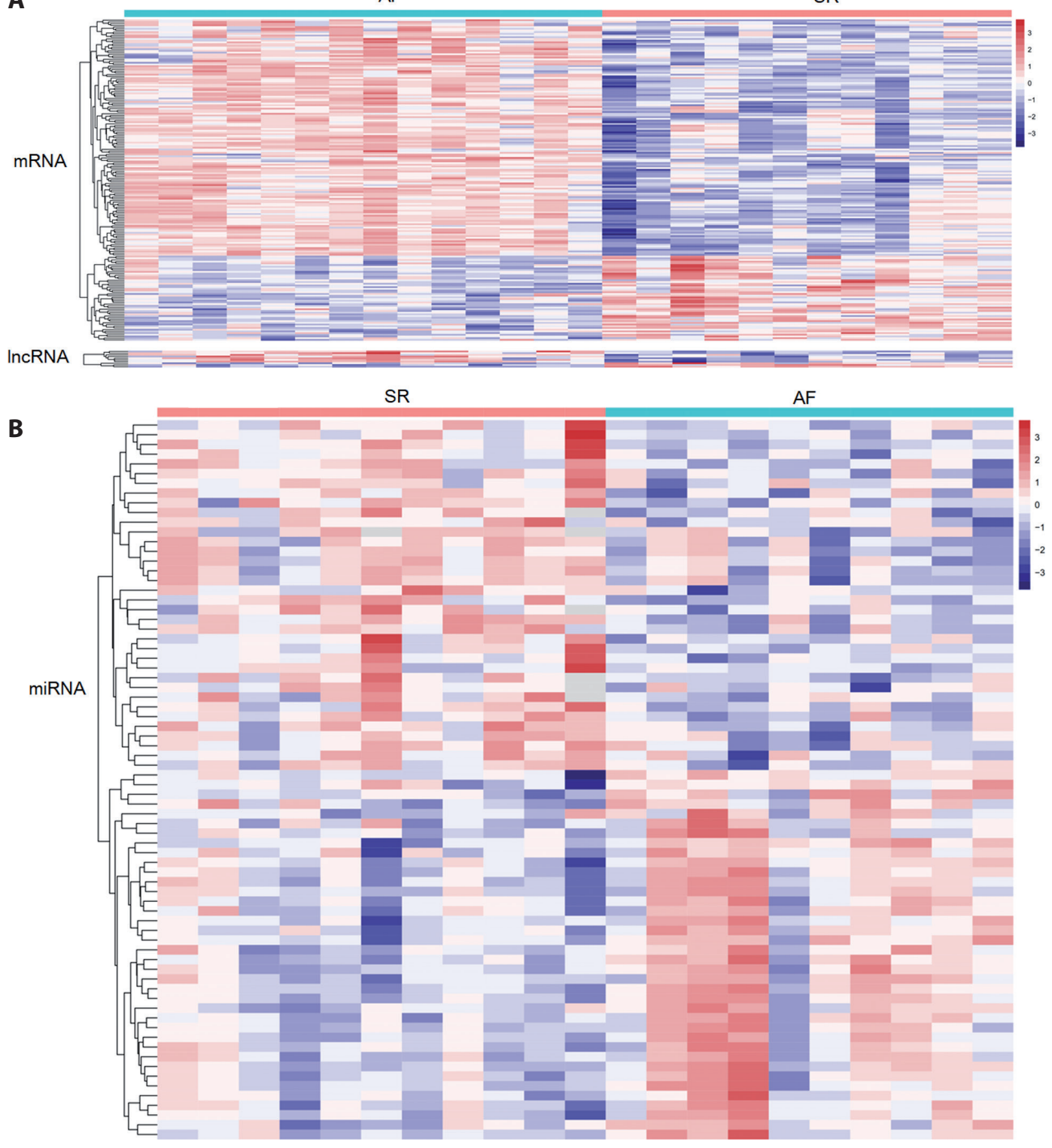

Figure 2. Identification of differentially expressed mRNAs, IncRNAs and miRNAs. A. The heat map of differentially expressed mRNAs and lncRNAs identified in the GSE79768 dataset. B. The heat map of differentially expressed miRNAs identified in the GSE68475 dataset. AF, atrial fibrillation; SR, sinus rhythm. Red, upregulated genes; blue, downregulated genes. 
miRNAMap, PicTar2, PITA, RNA22v2, RNAhybrid2.1 and Targetscan6.2) were selected to predict the DEMs-mRNA interaction pairs and the results predicted by more than 5 databases were retained. A total of 4 prediction programs (miRWalk, miRanda, RNAhybrid and Targetscan) were used to explore the DEMs-lncRNA relation pairs. The DEM-lncRNA interactions predicted by anyone database were enrolled. The lncRNAs and mRNAs that interacted with DEMs were then overlapped with the module DELs and DEGs. Also, the expression of module DELs and DEGs should be opposite to that of DEMs. The ceRNA network was constructed by Cytoscape using the finally left DEM-DEL and DEM-DEG interaction pairs.

\section{Function enrichment analysis}

The Gene Ontology (GO) terms and Kyoto Encyclopedia of Genes and Genomes (KEGG) pathway enrichment results of module DEGs were obtained by searching the online Database for Annotation, Visualization and Integrated Discovery (DAVID) database (version 6.8; http://david.abcc.ncifcrf. gov) (Dennis et al. 2003) and using the function enrichment algorithm of WGCNA procedure. $p$-value (or adjusted) $<0.05$ indicated the statistical significance.

\section{Prediction of potential drugs for the treatment of $A F$}

The upregulated and downregulated module DEGs were defined as query signatures to search the Connectivity Map (CMap) database (https://portals.broadinstitute.org/ $\mathrm{cmap} /$ ) to discover potential therapeutic drugs (negative connectivity score and $p$-value < 0.05) for AF. Furthermore, the Comparative Toxicogenomics Database (CTD; http:// ctdbase.org) was also searched to obtain the drug-lncRNA interaction pairs.

Table 2. Preserved modules

\begin{tabular}{|c|c|c|c|c|c|c|c|c|}
\hline \multirow{2}{*}{ Module } & \multirow{2}{*}{ Color } & \multirow{2}{*}{ Module size } & \multicolumn{2}{|c|}{ Preservation } & \multirow{2}{*}{ DEGs } & \multirow{2}{*}{ DELs } & \multicolumn{2}{|l|}{ Enrichment } \\
\hline & & & Z-score & $p$-value & & & Enrichment fold[95\%CI] & Phyper \\
\hline 1 & Black & 602 & 34.12 & $1.071 \mathrm{E}-341$ & 6 & 1 & $1.29[0.51-2.73]$ & $5.08 \mathrm{E}-01$ \\
\hline 2 & Blue & 2921 & 27.53 & $9.55 \mathrm{E}-209$ & 2 & - & $0.07[0.01-0.24]$ & $3.41 \mathrm{E}-10$ \\
\hline 3 & Brown & 1706 & 43.75 & $6.31 \mathrm{E}-419$ & 45 & 5 & $4.04[2.86-5.63]$ & $7.79 \mathrm{E}-14$ \\
\hline 4 & Cyan & 338 & 15.26 & $3.24 \mathrm{E}-55$ & & 2 & $0.64[0.08-2.38]$ & $7.73 \mathrm{E}-01$ \\
\hline 5 & Darkgreen & 151 & 11.93 & $1.35 \mathrm{E}-41$ & - & - & - & - \\
\hline 6 & Darkgrey & 134 & 11.34 & $8.13 \mathrm{E}-31$ & - & - & - & - \\
\hline 7 & Darkorange & 95 & 7.67 & $2.00 \mathrm{E}-15$ & - & - & - & - \\
\hline 8 & Darkred & 151 & 9.15 & $4.57 \mathrm{E}-25$ & - & - & - & - \\
\hline 9 & Darkturquoise & 141 & 9.89 & $1.10 \mathrm{E}-23$ & - & - & - & - \\
\hline 10 & Green & 788 & 10.68 & $2.95 \mathrm{E}-30$ & 11 & - & $1.57[0.77-2.89]$ & $1.75 \mathrm{E}-01$ \\
\hline 11 & Greenyellow & 479 & 19.41 & $1.82 \mathrm{E}-90$ & - & - & - & - \\
\hline 12 & Grey & 2069 & 12.6 & $3.24 \mathrm{E}-54$ & 4 & - & $0.19[0.05-0.51]$ & $4.39 \mathrm{E}-05$ \\
\hline 13 & Grey60 & 302 & 16.65 & $1.58 \mathrm{E}-80$ & 2 & - & $0.72[0.09-2.67]$ & $1.00 \mathrm{E}+00$ \\
\hline 14 & Lightcyan & 312 & 8.85 & $3.16 \mathrm{E}-19$ & 12 & 1 & $4.99[2.58-8.87]$ & $6.18 \mathrm{E}-06$ \\
\hline 15 & Lightgreen & 273 & 15.87 & $5.62 \mathrm{E}-60$ & - & - & - & - \\
\hline 16 & Lightyellow & 265 & 15.34 & $1.55 \mathrm{E}-66$ & - & - & - & - \\
\hline 17 & Magenta & 521 & 15.91 & $2.29 \mathrm{E}-61$ & 29 & - & $7.33[4.71-11.04]$ & $6.01 E-15$ \\
\hline 18 & Midnightblue & 335 & 15.67 & $3.16 \mathrm{E}-57$ & - & - & - & - \\
\hline 19 & Orange & 123 & 11.44 & $1.95 \mathrm{E}-40$ & - & - & - & - \\
\hline 20 & Pink & 561 & 15.1 & $2.95 \mathrm{E}-69$ & 8 & 1 & $1.81[0.81-3.53]$ & $1.07 \mathrm{E}-01$ \\
\hline 21 & Purple & 508 & 17.07 & $2.88 \mathrm{E}-83$ & 2 & - & $0.42[0.05-1.56]$ & $3.38 \mathrm{E}-01$ \\
\hline 22 & Red & 608 & 12.28 & $1.95 \mathrm{E}-36$ & 1 & - & $0.18[0-0.99]$ & $4.79 \mathrm{E}-02$ \\
\hline 23 & Royalblue & 192 & 11.63 & $4.27 \mathrm{E}-34$ & 1 & - & $0.57[0.01-3.24]$ & $1.00 \mathrm{E}+00$ \\
\hline 24 & Salmon & 378 & 5.72 & $1.05 \mathrm{E}-09$ & 3 & - & $0.87[0.18-2.6]$ & $1.00 \mathrm{E}+00$ \\
\hline 25 & Tan & 469 & 13.95 & $1.78 \mathrm{E}-60$ & 13 & - & $3.25[1.68-5.75]$ & $3.88 \mathrm{E}-04$ \\
\hline 26 & Turquoise & 6249 & 30.49 & $3.98 \mathrm{E}-251$ & 29 & 1 & $0.44[0.29-0.65]$ & $8.69 \mathrm{E}-06$ \\
\hline 27 & Yellow & 984 & 11.79 & $4.57 \mathrm{E}-43$ & 19 & 1 & $2.4[1.42-3.83]$ & 7.94E-04 \\
\hline
\end{tabular}

DEGs, differentially expressed mRNAs; DELs, differentially expressed lncRNAs; CI, confidence intervals. Bold were preserved modules and DEGs/DELs significantly enriched modules. 


\section{Results \\ Identification of DELs, DEGs and DEMs in AF}

In the analysis of the GSE79768 dataset, 1,056 mRNAs (831 upregulated and 225 downregulated) and 38 lncRNAs (24 upregulated and 14 downregulated) were found to be significantly differentially expressed in left atrial appendages of AF patients compared with SR ones; 313 DEGs (174 upregulated and 139 downregulated) and 17 DELs (9 upregulated and 8 downregulated) were identified in right atrial tissues between AF and SR patients. There were 10 common DELs and 189 common DEGs between left and right atrial tissues (Table 1). A total of 38 upregulated and 36 downregulated DEMs were identified between AF and SR in the GSE68475 dataset (Table 1). The heat maps of common DELs, DEGs and DEMs are shown in Figure 2, from which we could see the AF samples were obviously separated from SR ones.

\section{Identification of AF-associated modules}

As shown in Figure 2, the expression ( $\mathrm{r}=0.92$; Fig. 3A) and connectivity ( $r=0.27$; Fig. $3 \mathrm{~B}$ ) of 21,655 RNAs were significantly positively correlated in the GSE79768 and GSE115574 datasets ( $p$-value $<1.00 \mathrm{E}-200$ ), indicating they were comparable. The soft-thresholding power of $\beta$ was selected as 8 to fit a scale-free network (scale-free $\mathrm{R}^{2}>0.90$, Fig. $3 \mathrm{C}$; mean connectivity $=1$, Fig. 3D). A total of 27 co-expression modules were screened from the GSE79768 dataset using the Dynamic TreeCut method (Fig. 4A; Table 2). These modules were also identified after analysis with the GSE115574 dataset (Figure 4B). Twenty-two of them were highly preserved $(\mathrm{Z}$-score $>10, p$-value $<0.05)$ and the other five were moderately preserved $(5<\mathrm{Z}$-score $<10$ and $p$-value $<0.05)$ in these two datasets. Mapping analysis with DELs and DEGs further suggested five modules (brown, lightcyan, magenta, tan and yellow) may be particularly important for the development of AF (Table 2). This hypothesis was shown to be believable because brown $(\mathrm{r}=-0.69, p=1.00 \mathrm{E}-04)$, lightcyan $(\mathrm{r}=-0.58, p=2.00 \mathrm{E}-03)$, magenta $(\mathrm{r}=-0.65, p=$ $3.00 \mathrm{E}-04), \tan (\mathrm{r}=-0.49, p=1.00 \mathrm{E}-02)$ and yellow $(\mathrm{r}=0.46$, $p=2.00 \mathrm{E}-02)$ modules were detected to be highly related to the clinical trait of AF status in module-trait relationship analysis (Fig. 4C). The gene significance of all these modules was $>0.3$ (Fig. 5A). The GS for AF status and MM in these five modules also showed a strongly significant correlation (Fig. 5B-F). These findings indicated genes in these modules were highly relevant with AF. Thus, 118 DEGs and 7 DELs in these five modules were used for the following analyses.

\section{Identification of hub DEGs in the modules}

A total of 90 DEGs in the above five modules had $|\mathrm{GS}|>0.6$ and $|\mathrm{MM}|>0.5$, suggesting they may be hub genes according

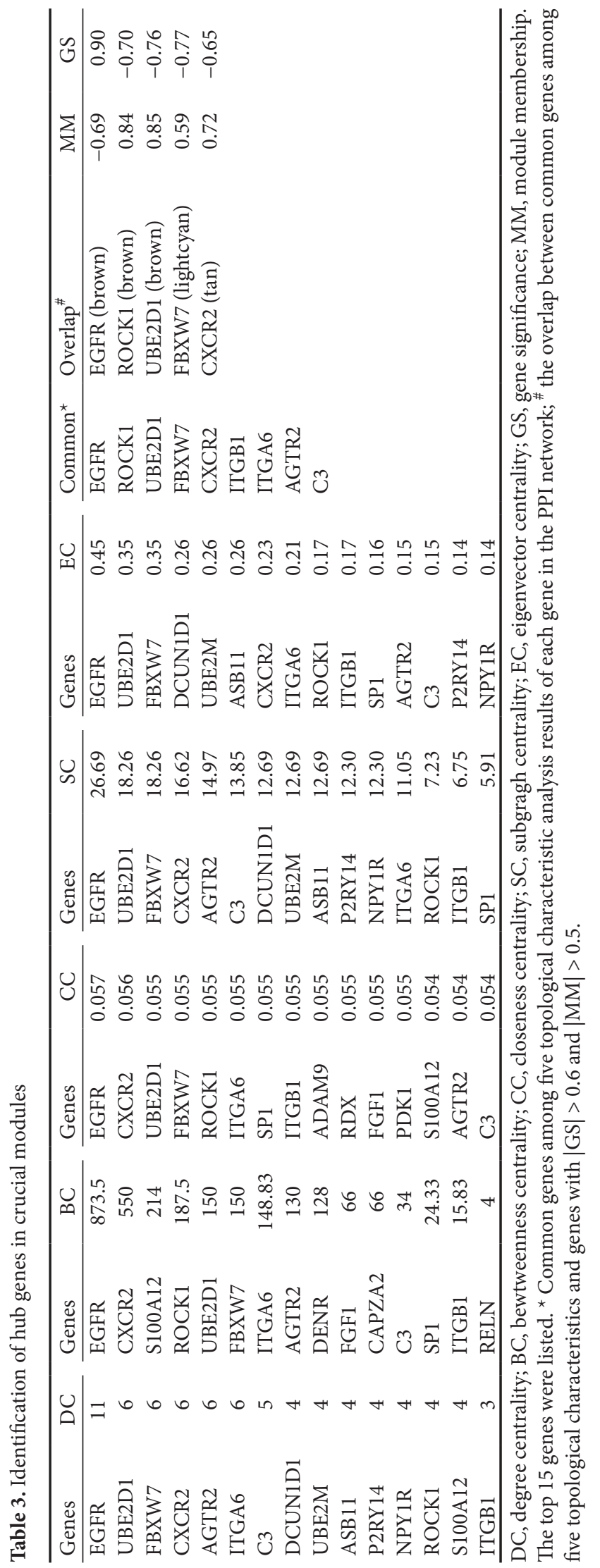


to WGCNA analysis. Identification of gene co-expression modules was purely based on mathematical correlations among genes in the individual module. To further explore potential biological associations among genes in these five modules and screen hub DEGs, we performed a PPI network analysis. As a result, STRING database analysis screened 65 PPI interaction pairs between 51 module DEGs, which were used for the construction of the PPI network (Fig. 6A). Among these 51 DEGs, 9 were considered to be hub genes because they ranked in the top 15 of all topological characteristics (DC, BC, CC, SC and EC) (Table 3). Furthermore,
A

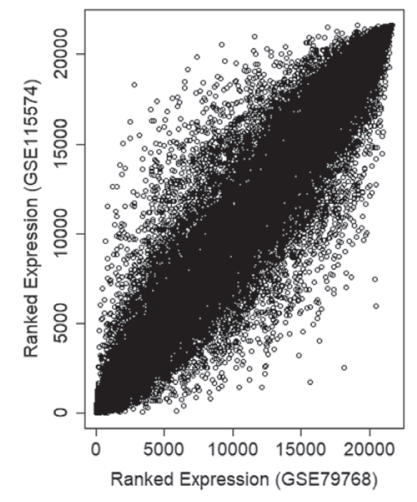

C
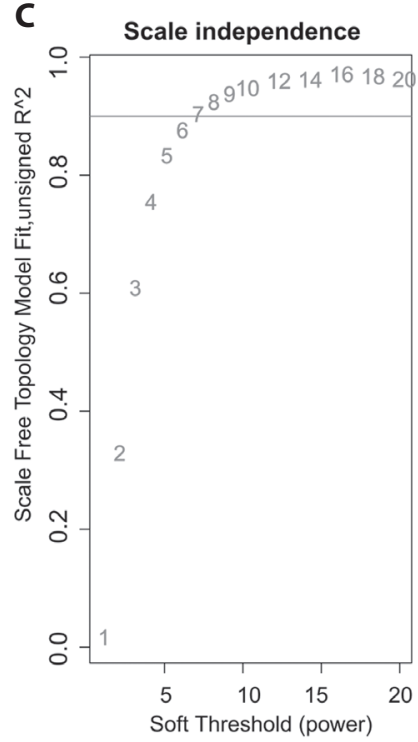

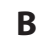

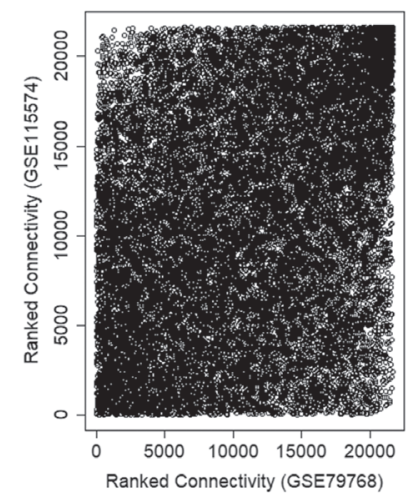

D

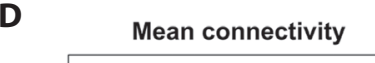

Figure 3. Assessment of the correlation between two datasets and determination of soft-threshold power $\beta$ for WGCNA (Weighted gene co-expression network analysis). A. The correlation of the RNA expression levels between GSE79768 and GSE115574 datasets $(\mathrm{r}=0.92, p<1.00 \mathrm{E}-200)$. B. The correlation of the connectivity between GSE79768 and GSE115574 datasets ( $r=0.27$, $p<1.00 \mathrm{E}-200)$. C. Analysis of the scale-free fit index for various $\beta$ values (from 1-20). D. Analysis of the mean connectivity for each $\beta$ value. the hub genes identified by WGCNA and PPI were compared and the results showed five genes were overlapped (Fig. 6B; Table 3), including ROCK1 (Rho associated coiled-coil containing protein kinase 1), EGFR (epidermal growth factor receptor), CXCR2 (C-X-C motif chemokine receptor 2), FBXW7 (F-box and WD repeat domain containing 7) and UBE2D1 (ubiquitin conjugating enzyme E2 D1). Except for ROCK1 (only expressed in male samples), the other four genes were differentially expressed in both female and male samples. Also, the false discovery rate (FDR) of these genes was $<0.05$ in left atrial samples of AF patients (Table 1). These findings demonstrated the importance of these five hub genes for AF.

\section{Function analysis for hub genes}

To obtain the functions of hub genes, 125 DEGs in the crucial modules were uploaded into the DAVID database. As a result, 13 significant GO biological process terms and 4 significant GO molecular function terms were enriched (Table 4, $p<0.05$ ), such as GO:1902806 regulation of cell cycle G1/S phase transition (FBXW7), GO:0030335 positive regulation of cell migration (EGFR), GO:0006954 inflammatory response (CXCR2) and GO:0050900 leukocyte migration (ROCK1). Moreover, KEGG pathway analysis revealed that the module genes were associated with regulation of actin cytoskeleton (ROCK1, EGFR) and focal adhesion (ROCK1, EGFR) (Table 4). In addition, we also performed function enrichment analysis for genes in each module by WGCNA to reveal their roles. According to the threshold of adjusted $p$-value $<0.05$, UBE2D1 and CXCR2 were shown to be related with proteasome-mediated ubiquitin-dependent protein catabolic process and Cytokine-cytokine receptor interaction, respectively.

\section{Identification of hub lncRNAs that regulated hub DEGs}

Co-expression and ceRNA networks were constructed to identify hub module lncRNAs. A total of 398 co-expression relationship pairs between seven module DELs and 107 module DEGs had $\mathrm{r}>0.5$ and $p$-value $<0.01$; thus, they were used to establish the lncRNA-mRNA co-expression network (Fig. 7). Among them, seven lncRNAs (MIR100HG, LOC101928304, USP3-AS1, LINC01105, LINC02502, TRDN-AS1, RP11$420 \mathrm{~K} 14.2$ ) were shown to positively regulate the expressions of five hub genes (ROCK1, EGFR, FBXW7, CXCR2, UBE2D1) identified by WGCNA and PPI network analyses.

Twenty-six DEMs were predicted to commonly interact with three module DELs and 71 module DEGs. Thus, they were used to establish the IncRNA-miRNA-mRNA ceRNA network (Fig. 8). Among them, five hub genes (ROCK1, EGFR, CXCR2, FBXW7, UBE2D1) identified by WGCNA and PPI network analysis were found to be regulated by three DELs (MIR100HG, 
LINC01105, USP3-AS1) and 12 miRNAs (hsa-miR-4314, hsamiR-3658, hsa-miR-1183, hsa-miR-4257, hsa-miR-200b-3p, hsa-miR-3926, hsa-miR-548z, hsa-miR-3692-5p, hsa-miR125a-3p, hsa-miR-323a-5p, hsa-miR-573, hsa-miR-561-3p).

A comparison of the co-expression and ceRNA regulatory pairs indicated that LINC01105 and MIR100HG with FDR $<0.05$ in left atrial samples of AF patients (both females and males) (Table 1) may be particularly crucial lncRNAs because they regulated the expression of the same hub genes via both the co-expression and the ceRNA mechanisms, including LINC01105-miR-125a-3p-EGFR, LINC01105EGFR, MIR100HG-miR-200b-3p-FBXW7, MIR100HGFBXW7, MIR100HG-miR-548z-UBE2D1 and MIR100HGUBE2D1. Furthermore, MIR100HG-miR-561-3p-CXCR2 and MIR100HG-ROCK1 interaction axes may also be vital.

\section{Identification of potential small molecule drugs for AF}

The 93 upregulated module DEGs and 25 downregulated module DEGs were used as the query signature for the
A

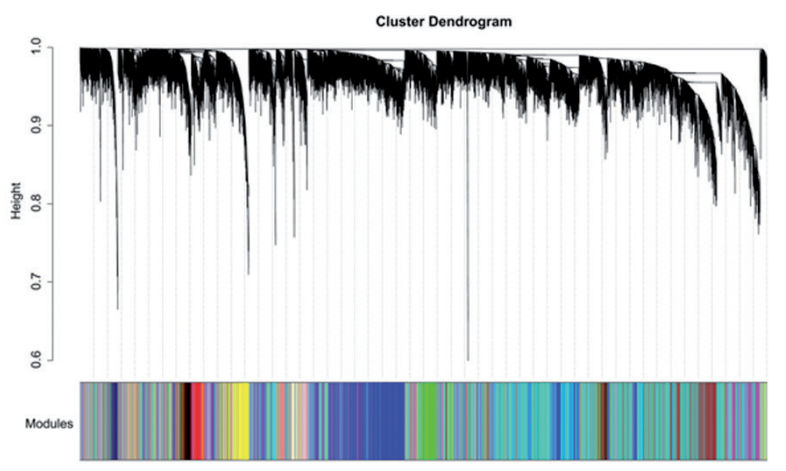

B

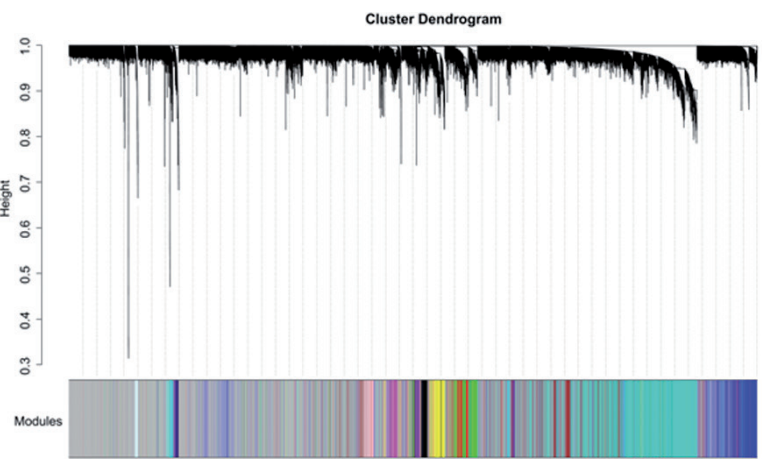

C

Module-trait relationships

\begin{tabular}{|c|c|c|c|c|}
\hline MElightyellow & $r=0.034 P=0.9$ & $r=-0.42 P=0.03$ & $r=0.21 P=0.3$ & $r=0.48 P=0.01$ \\
\hline MEcyan & $r=0.016 P=0.9$ & $r=0.33 P=0.1$ & $r=0.41 P=0.04$ & $r=0.26 P=0.2$ \\
\hline MEsalmon & $r=0.24 P=0.2$ & $r=0.3 P=0.1$ & $r=0.41 P=0.04$ & $r=0.61 P=0.001$ \\
\hline MElightgreen & $r=-0.062 P=0.8$ & $r=0.37 P=0.07$ & $r=-0.055 P=0.8$ & $r=0.18 P=0.4$ \\
\hline MEdarkgreen & $r=0.18 \mathrm{P}=0.4$ & $r=0.21 P=0.3$ & $r=-0.31 P=0.1$ & $r=-0.39 P=0.05$ \\
\hline MEgreenyellow & $r=0.27 P=0.2$ & $r=0.19 P=0.4$ & $r=-0.34 P=0.09$ & $r=-0.34 P=0.08$ \\
\hline MEblack & $r=0.23 P=0.3$ & $r=-0.12 P=0.6$ & $r=-0.66 P=3 e-04$ & $r=-0.5 P=0.009$ \\
\hline MElightcyan & $r=0.25 P=0.2$ & $r=-0.044 P=0.8$ & $r=-0.37 P=0.06$ & $r=-0.58 P=0.002$ \\
\hline MEmagenta & $r=0.14 P=0.5$ & $r=0.23 P=0.3$ & $r=-0.26 P=0.2$ & $r=-0.65 P=3 e-04$ \\
\hline MEbrown & $r=0.15 P=0.5$ & $r=0.38 P=0.06$ & $r=-0.19 P=0.3$ & $r=-0.69 P=1 e-04$ \\
\hline MEdarkred & $r=0.14 P=0.5$ & $r=0.48 P=0.01$ & $r=0.043 P=0.8$ & $r=-0.34 P=0.09$ \\
\hline MEdarkgrey & $r=0.031 P=0.9$ & $r=-0.06 P=0.8$ & $r=-0.25 P=0.2$ & $r=0.068 P=0.7$ \\
\hline MEyellow & $r=-0.51 P=0.008$ & $r=0.07 P=0.7$ & $r=0.26 P=0.2$ & $r=0.46 P=0.02$ \\
\hline MEgreen & $r=0.028 P=0.9$ & $r=-0.14 P=0.5$ & $r=0.074 P=0.7$ & $r=0.53 P=0.005$ \\
\hline MEorange & $r=0.25 P=0.2$ & $r=-0.27 P=0.2$ & $r=-0.39 P=0.05$ & $r=0.24 P=0.2$ \\
\hline MEred & $r=0.16 P=0.4$ & $r=-0.43 P=0.03$ & $r=-0.49 P=0.01$ & $r=-0.25 P=0.2$ \\
\hline MEdarkturquoise & $r=0.13 P=0.5$ & $r=-0.25 P=0.2$ & $r=-0.3 P=0.1$ & $r=-0.23 P=0.2$ \\
\hline MEpurple & $r=-6 e-04 P=1$ & $r=-0.4 P=0.04$ & $r=-0.38 P=0.06$ & $r=-0.43 P=0.03$ \\
\hline MEdarkorange & $r=0.013 P=0.9$ & $r=-0.84 P=7 e-08$ & $r=-0.52 P=0.006$ & $r=-0.047 P=0.8$ \\
\hline MEroyalblue & $r=-0.18 P=0.4$ & $r=-0.5 P=0.009$ & $r=-0.24 P=0.2$ & $r=-0.13 P=0.5$ \\
\hline MEblue & $r=-0.29 P=0.2$ & $r=-0.62 P=8 e-04$ & $r=-0.28 P=0.2$ & $r=-0.22 P=0.3$ \\
\hline MEturquoise & $r=-0.094 P=0.6$ & $r=-0.56 P=0.003$ & $r=-0.019 P=0.9$ & $r=0.44 P=0.03$ \\
\hline MEmidnightblue & $r=-0.18 P=0.4$ & $r=-0.025 P=0.9$ & $r=0.096 P=0.6$ & $r=0.18 P=0.4$ \\
\hline MEpink & $r=-0.14 P=0.5$ & $r=0.034 P=0.9$ & $r=-0.13 P=0.5$ & $r=-0.57 P=0.002$ \\
\hline MEgrey60 & $r=-0.63 P=6 e-04$ & $r=-0.34 P=0.09$ & $r=-0.33 P=0.1$ & $r=-0.27 P=0.2$ \\
\hline MEtan & $r=-0.66 P=2 e-04$ & $r=0.0085 P=1$ & $r=-0.27 P=0.2$ & $r=-0.49 P=0.01$ \\
\hline MEgrey & $r=-0.17 P=0.4$ & $r=-0.31 P=0.1$ & $r=-0.04 P=0.8$ & $r=0.34 P=0.09$ \\
\hline
\end{tabular}

Figure 4. Identification of modules associated with atrial fibrillation. A. Dendrogram of lncRNAs and mRNAs in the GSE79768 dataset. B. Dendrogram of lncRNAs and mRNAs in the GSE115574 dataset. C. Heat map to show the correlation between module eigengenes and the clinical traits of atrial fibrillation patients. The left color scale is corresponding to each module. The right color scale indicates the association. Red, positive associations; blue, negative associations. 
A

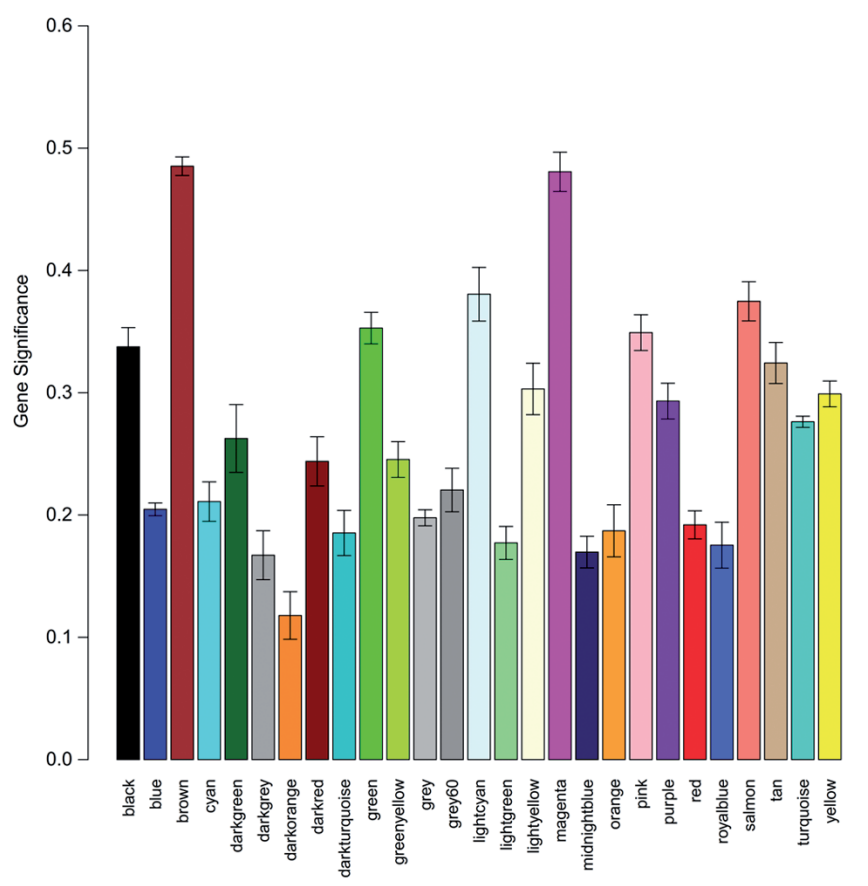

B

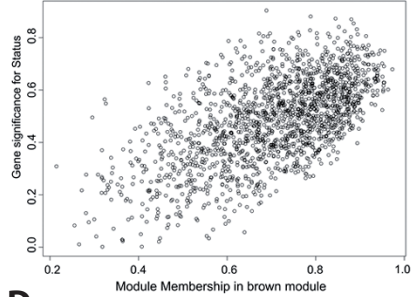

D

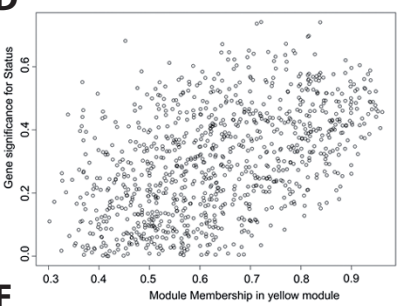

F

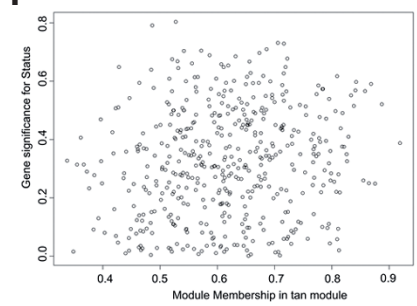

C

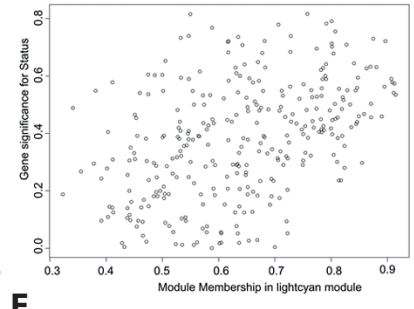

E

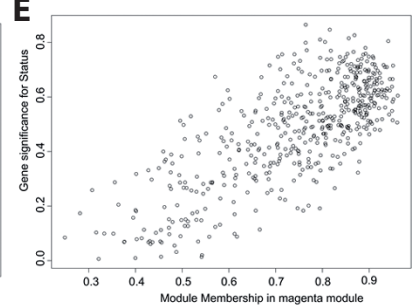

Figure 5. The significance of genes in identified modules. A. Distribution of atrial fibrillation-related genes in all modules. Gene significance across modules $(p=0)$. The correlation plot of gene significance versus module membership for genes contained in brown $(\mathrm{r}=0.61, p=2.00 \mathrm{E}-174 ; \mathrm{B})$, lightcyan $(\mathrm{r}=0.44, p=3.30 \mathrm{E}-16 ; \mathrm{C})$, yellow $(\mathrm{r}=0.49, p=1.50 \mathrm{E}-60 ; \mathrm{D})$, magenta $(\mathrm{r}=0.75, p=3.20 \mathrm{E}-95$; E) and $\tan (\mathrm{r}=0.149, p=2.40 \mathrm{E}-3 ; \mathrm{F})$ modules.

CMap database to retrieve potential drugs for the treatment of AF. A total of 57 compounds with the negative enrichment score and $p$-value $<0.05$ were obtained, such as artemisinin (Table 5). These small molecule drugs may exert a similar function to LINC01105 overexpression or anti-MIR100HG to reverse the expression of hub
A

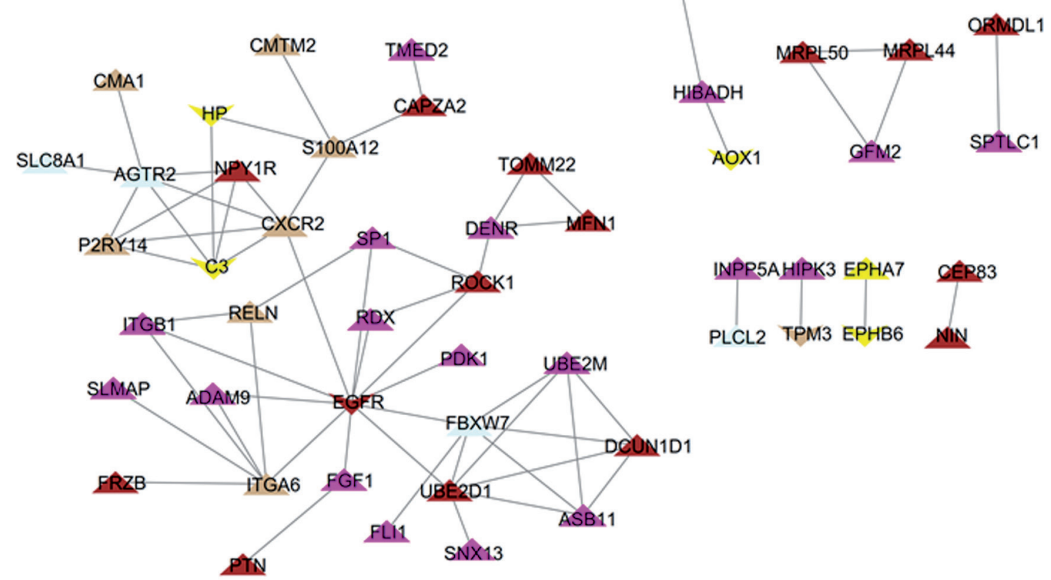

B

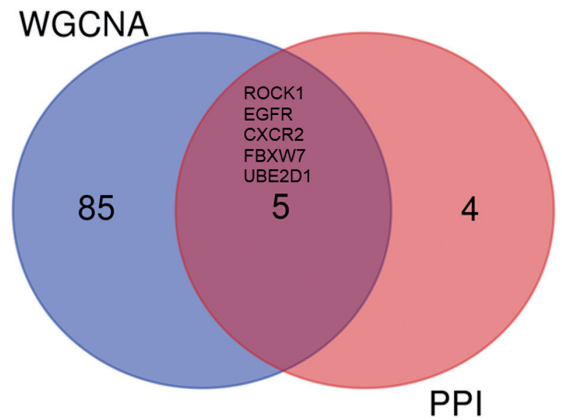

Figure 6. Identification of hub genes in modules. A. Construction of the PPI network for all the differentially expressed mRNAs in five preserved modules. Different colors represent corresponding modules. B. The overlap between common genes among five topological characteristics of PPI network and genes with $|\mathrm{GS}|>0.6$ and $|\mathrm{MM}|>0.5$ in WGCNA. WGCNA, weighted gene co-expression network analysis; PPI, protein-protein interaction; GS, gene significance; MM, module membership. 
DEGs. Furthermore, the interactions between lncRNAs and chemicals were predicted by CTD analysis. As a result, dexamethasone was found to be a targeted drug for MIR100HG by decreasing its mRNA expression, indicating dexamethasone may be an underlying drug for the treatment of AF.

\section{Discussion}

Based on WGCNA, IncRNA-mRNA co-expression and lncRNA-miRNA-mRNA ceRNA network analyses, our study identified MIR100HG and LINC01105 significantly associated with the development of AF and they may represent

Table 4. Function enrichment analysis for the genes in the crucial modules

\begin{tabular}{|c|c|c|c|c|}
\hline Category & ID & Term & $\begin{array}{c}p \text {-value } \\
\text { (or adjusted) }\end{array}$ & Genes \\
\hline \multirow{13}{*}{$\begin{array}{l}\text { GO BP } \\
\text { (DAVID) }\end{array}$} & GO:0033627 & Cell adhesion mediated by integrin & $3.71 \mathrm{E}-03$ & ITGB1, ADAM9, ITGA6 \\
\hline & GO:0042327 & Positive regulation of phosphorylation & $1.02 \mathrm{E}-02$ & MOB1B, ITGA6, EGFR \\
\hline & GO:0033631 & $\begin{array}{l}\text { Cell-cell adhesion mediated by } \\
\text { integrin }\end{array}$ & $2.43 \mathrm{E}-02$ & ITGB1, ADAM9 \\
\hline & GO:1902806 & $\begin{array}{l}\text { Regulation of cell cycle G1/S phase } \\
\text { transition }\end{array}$ & $2.43 \mathrm{E}-02$ & FBXW7, C8ORF4 \\
\hline & GO:0030335 & Positive regulation of cell migration & $2.67 \mathrm{E}-02$ & FAM110C, RDX, ITGA6, FGF1, EGFR \\
\hline & GO:0043525 & $\begin{array}{l}\text { Positive regulation of neuron } \\
\text { apoptotic process }\end{array}$ & $2.86 \mathrm{E}-02$ & ATF2, EPHA7, UBE2M \\
\hline & GO:0006954 & Inflammatory response & $2.92 \mathrm{E}-02$ & AIMP1, C3, CXCR2, S100A12, AOX1, AGTR2, BMPR1B \\
\hline & GO:0031952 & $\begin{array}{l}\text { Regulation of protein } \\
\text { autophosphorylation }\end{array}$ & $3.03 \mathrm{E}-02$ & MOB1B, EPHA7 \\
\hline & GO:0008015 & Blood circulation & $3.11 \mathrm{E}-02$ & RCAN1, NPY1R, FLI1 \\
\hline & GO:0045766 & Positive regulation of angiogenesis & $3.38 \mathrm{E}-02$ & C3, CMA1, CXCR2, FGF1 \\
\hline & GO:0042542 & Response to hydrogen peroxide & $3.91 \mathrm{E}-02$ & HP, ADAM9, SLC8A1 \\
\hline & GO:0050900 & Leukocyte migration & $3.92 \mathrm{E}-02$ & AIMP1, ITGB1, ROCK1, ITGA6 \\
\hline & GO:0042698 & Ovulation cycle & $4.80 \mathrm{E}-02$ & BMPR1B, EGFR \\
\hline \multirow[t]{2}{*}{$\begin{array}{l}\text { GO BP } \\
\text { (WGCNA) }\end{array}$} & $\begin{array}{l}\text { GO:0043161 } \\
\text { (brown) }\end{array}$ & $\begin{array}{l}\text { Proteasome-mediated ubiquitin- } \\
\text { dependent protein catabolic process }\end{array}$ & $1.37 \mathrm{E}-04$ & $\begin{array}{l}\text { ANAPC10, ANAPC16 ANAPC2, ARAF, BAG5, BUB3, } \\
\text { CCDC47, CDC23, CUL2, CUL4A, CUL5, DERL1, } \\
\text { DNAJB9, FAF1, FBXL17, FBXL6, FBXO5, GCLC, } \\
\text { IL33, JKAMP, LRRK2, MAD2L1, NEDD4, NEMF, } \\
\text { OGT, PIAS1, PLAA, PLK1, PSMA4, PSMB3, PSMB5, } \\
\text { PSMC2, PSMC6, PSMD7, PSME4, RAD23B RBX1, } \\
\text { RMND5A, RNF187, RPS27A, SDCBP, SIRT1, SIRT6, } \\
\text { SKP1, SUMO1, TBL1XR1, TMUB1, TOPORS, UBB, } \\
\text { UBE2A, UBE2B, UBE2D1, UBE2D3, UBE2W, UBR1, } \\
\text { UCHL5, UFL1 }\end{array}$ \\
\hline & $\begin{array}{l}\text { GO:0051480 } \\
(\tan )\end{array}$ & $\begin{array}{l}\text { Regulation of cytosolic calcium ion } \\
\text { concentration }\end{array}$ & $5 . .60 \mathrm{E}-04$ & $\begin{array}{l}\text { ADM, AVPR1A, C1QTNF1, CAV2, CCL21, CD36, } \\
\text { CNR1, CXCR1, CXCR2, CXCR6, CYSLTR1, FKBP1B, } \\
\text { GJA1, JPH1, KCNA5, MCOLN3, P2RY8, PLCG2, } \\
\text { PTGDR, PTGFR, SCGN, TBXA2R, TRPC6 }\end{array}$ \\
\hline \multirow{4}{*}{$\begin{array}{l}\text { KEGG } \\
\text { (DAVID) }\end{array}$} & hsa04810 & Regulation of actin cytoskeleton & $9.47 \mathrm{E}-03$ & ITGB1, ROCK1, RDX, ITGA6, FGF1, EGFR \\
\hline & hsa05410 & Hypertrophic cardiomyopathy (HCM) & $1.25 \mathrm{E}-02$ & ITGB1, TPM3, PRKAG2, ITGA6 \\
\hline & hsa04510 & Focal adhesion & $3.88 \mathrm{E}-02$ & ITGB1, RELN, ROCK1, ITGA6, EGFR \\
\hline & hsa04360 & Axon guidance & $4.44 \mathrm{E}-02$ & EPHB6, ITGB1, EPHA7, ROCK1 \\
\hline \multirow[t]{2}{*}{$\begin{array}{l}\text { KEGG } \\
\text { (WGCNA) }\end{array}$} & $\begin{array}{l}\text { hsa04120 } \\
\text { (brown) }\end{array}$ & Ubiquitin mediated proteolysis & $1.86 \mathrm{E}-03$ & $\begin{array}{l}\text { ANAPC10, ANAPC2, CBLB, CDC23, CUL2, CUL4A, } \\
\text { CUL5, FANCL, NEDD4, PIAS1, PRPF19, RBX1, } \\
\text { RPS27A, SKP1, UBA1, UBA3, UBB, UBE2A, UBE2B, } \\
\text { UBE2D1, UBE2D3, UBE2D4, UBE2E2, UBE2W }\end{array}$ \\
\hline & $\begin{array}{l}\text { hsa04060 } \\
(\tan )\end{array}$ & $\begin{array}{l}\text { Cytokine-cytokine receptor } \\
\text { interaction }\end{array}$ & $1.13 \mathrm{E}-02$ & $\begin{array}{l}\text { BMP10, BMPR1B, CCL21, CXCR1, CXCR2, CXCR6, } \\
\text { EDA, IL12RB2, IL13RA2, IL17RB, IL4R, INHA, INHBB, } \\
\text { LEPR, NGF, TNFRSF10B, TNFRSF10C, TNFRSF11B }\end{array}$ \\
\hline
\end{tabular}


potential therapeutic targets for the blockade of AF. Upregulated MIR100HG could regulate the transcription of all significantly upregulated DEGs (FBXW7, UBE2D1, CXCR2, ROCK1); downregulated LINC01105 may function by inducing the low expression of EGFR via co-expression and/ or ceRNA mechanisms. FBXW7 was involved in regulation of cell cycle; ROCK1 and EGFR may mediate the cell migration and adhesion; CXCR2 participated in inflammatory processes; UBE2D1 was related with ubiquitin-dependent protein catabolic process.

Current studies of MIR100HG (Huang et al. 2019; Chen et al. 2020) and LINC01105 (Tang et al. 2016; Ye et al. 2019) mainly focused on their roles in tumorigenesis; while no reports explored their functions in cardiovascular diseases, indicating they may be new targets for AF. However, some downstream genes identified in our co-expression or ceRNA networks (including CXCR2, ROCK1, FBXW7, EGFR) had been demonstrated to be associated with the phenotypes of AF or known pathogenesis of AF (such as inflammation (Hu et al. 2015), cardiomyocyte apoptosis (Zhang and Jing 2018), atrial fibroblast proliferation to induce atrial fibrosis (Cao et al. 2019)), which may indirectly reflect their possible mechanisms in AF. For example, Zhang et al. (2020a) provided evidence that the expression level of CXCR2 and the number of CXCR2+ immune cells were markedly increased in the atria of angiotensin (Ang) II-infusion-induced AF models. The administration of CXCR2 inhibitor SB225002 or knockout of CXCR2 significantly reduced AF inducibility, duration, conduction abnormalities and atrial fibrosis in mice compared with the vehicle treatment (Zhang et al. 2020a, 2020b). Fan and Wei (2020) and Zou et al. (2019) also identified upregulated CXCR2 as a hub node for AF patients after the PPI network analysis of the GSE79768 dataset. The study results of Chen et al. showed that the expression of ROCK1 was significantly upregulated in left atrial append-

Table 5. CMap enrichment results

\begin{tabular}{|c|c|c|c|c|c|}
\hline Cmap name & Enrichment & $p$-value & Cmap name & Enrichment & $p$-value \\
\hline MS-275 & -0.939 & $7.67 \mathrm{E}-03$ & bendroflumethiazide & -0.672 & $3.38 \mathrm{E}-03$ \\
\hline 5252917 & -0.912 & $1.54 \mathrm{E}-02$ & crotamiton & -0.670 & $2.66 \mathrm{E}-02$ \\
\hline sanguinarine & -0.901 & $1.97 \mathrm{E}-02$ & 6-azathymine & -0.657 & $3.15 \mathrm{E}-02$ \\
\hline promethazine & -0.866 & $6.20 \mathrm{E}-04$ & harmine & -0.653 & $3.39 \mathrm{E}-02$ \\
\hline etomidate & -0.834 & $9.15 \mathrm{E}-03$ & propantheline bromide & -0.646 & $3.76 \mathrm{E}-02$ \\
\hline rimexolone & -0.825 & $1.79 \mathrm{E}-03$ & sulfametoxydiazine & -0.638 & $4.10 \mathrm{E}-02$ \\
\hline cortisone & -0.809 & $1.40 \mathrm{E}-02$ & ornidazole & -0.637 & $1.63 \mathrm{E}-02$ \\
\hline $\mathrm{N}$-acetyl-L-leucine & -0.805 & $2.80 \mathrm{E}-03$ & aminophylline & -0.637 & $4.17 \mathrm{E}-02$ \\
\hline methacholine chloride & -0.801 & $1.59 \mathrm{E}-02$ & propafenone & -0.633 & $4.42 \mathrm{E}-02$ \\
\hline GW-8510 & -0.792 & $3.80 \mathrm{E}-03$ & napelline & -0.629 & $4.62 \mathrm{E}-02$ \\
\hline hydrocotarnine & -0.790 & $3.98 \mathrm{E}-03$ & rolitetracycline & -0.629 & $4.63 \mathrm{E}-02$ \\
\hline lorglumide & -0.773 & $1.06 \mathrm{E}-03$ & Zimeldine & -0.626 & $1.91 \mathrm{E}-02$ \\
\hline trazodone & -0.768 & $2.57 \mathrm{E}-02$ & skimmianine & -0.626 & $4.81 \mathrm{E}-02$ \\
\hline verteporfin & -0.767 & $2.59 \mathrm{E}-02$ & piribedil & -0.626 & $4.83 \mathrm{E}-02$ \\
\hline doxorubicin & -0.752 & $3.09 \mathrm{E}-02$ & tyloxapol & -0.625 & $4.85 \mathrm{E}-02$ \\
\hline artemisinin & -0.738 & $3.66 \mathrm{E}-02$ & propofol & -0.624 & $4.95 \mathrm{E}-02$ \\
\hline Prestwick-559 & -0.733 & $3.89 \mathrm{E}-02$ & sulfafurazole & -0.622 & $2.01 \mathrm{E}-02$ \\
\hline repaglinide & -0.722 & $1.22 \mathrm{E}-02$ & cefalexin & -0.614 & $2.38 \mathrm{E}-02$ \\
\hline ginkgolide A & -0.712 & $1.39 \mathrm{E}-02$ & sulfadimidine & -0.613 & $1.08 \mathrm{E}-02$ \\
\hline fenoprofen & -0.706 & $1.57 \mathrm{E}-03$ & meclofenoxate & -0.611 & $1.15 \mathrm{E}-02$ \\
\hline dioxybenzone & -0.705 & $1.57 \mathrm{E}-02$ & phthalylsulfathiazole & -0.603 & $2.80 \mathrm{E}-02$ \\
\hline fluocinonide & -0.701 & $5.19 \mathrm{E}-03$ & dipyridamole & -0.599 & $1.45 \mathrm{E}-02$ \\
\hline sulfamethoxypyridazine & -0.699 & $5.41 \mathrm{E}-03$ & medrysone & -0.586 & $1.75 \mathrm{E}-02$ \\
\hline hexestrol & -0.691 & $1.94 \mathrm{E}-02$ & oleandomycin & -0.565 & $4.75 \mathrm{E}-02$ \\
\hline cetirizine & -0.687 & $2.07 \mathrm{E}-02$ & guaifenesin & -0.551 & $3.14 \mathrm{E}-02$ \\
\hline dacarbazine & -0.685 & $2.13 \mathrm{E}-02$ & famprofazone & -0.543 & $3.60 \mathrm{E}-02$ \\
\hline Cycloserine & -0.684 & $2.18 \mathrm{E}-02$ & cloperastine & -0.525 & $4.69 \mathrm{E}-02$ \\
\hline piromidic acid & -0.680 & $2.30 \mathrm{E}-02$ & felodipine & -0.498 & $3.81 \mathrm{E}-02$ \\
\hline amoxapine & -0.673 & $8.69 \mathrm{E}-03$ & & & \\
\hline
\end{tabular}



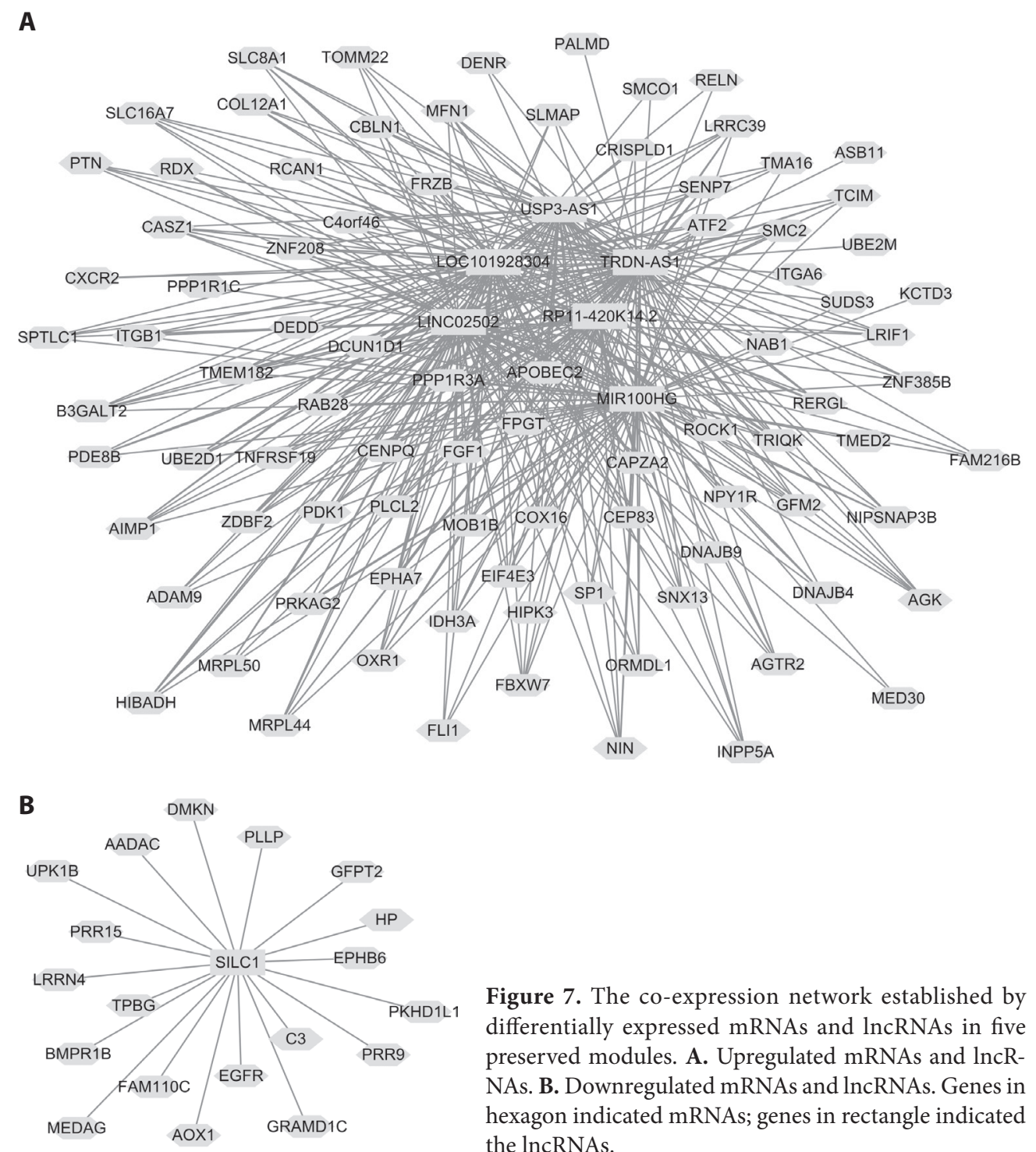

Figure 7. The co-expression network established by differentially expressed mRNAs and lncRNAs in five preserved modules. A. Upregulated mRNAs and lncRNAs. B. Downregulated mRNAs and lncRNAs. Genes in hexagon indicated mRNAs; genes in rectangle indicated the lncRNAs.

ages of patients with AF compared with those in the SR group. The association analysis indicated that ROCK1 may contribute to the pathogenesis of AF lesions by phosphorylation of MYPT-1 and then inducing the expression of connexin 40 (Chen et al. 2018). The expression of ROCK1 was found to be significantly higher in myolytic left atrial myocytes of mitral regurgitation AF patients than that of normal subjects. An immunofluorescence study revealed a significant co-localization of upregulated ROCK1 and pro-apoptotic cleaved caspase- 3 in atrial myocytes of AF patients (Chen et al. 2015). Liu et al. observed the mRNA expression level of ROCK1 in the left atrium of canine AF models and Ang II-treated atrial fibroblasts. The use of Rho-kinase inhibitor reduced the expression of ROCK1 and the proliferation of fibroblasts (Liu et al. 2016). Overex- pression of FBXW7 was reported to promote the apoptosis of mouse hearts and increase the infarct size (Chen et al. 2019b). From these findings, we speculated MIR100HG that positively regulated FBXW7, CXCR2 and ROCK1 expression may exert similar pro-apoptotic functions in AF. Anti-apoptotic and anti-inflammatory drugs may target MIR100HG followed by its downstream genes (or directly target mRNAs) to treat AF. In line with this hypothesis, we predicted dexamethasone was a MIR100HG-targeted, while artemisinin was a hub DEG-targeted drug for AF. Existing evidence had revealed that intraoperative treatment with dexamethasone could significantly reduce the release of several inflammatory factors (Yared et al. 2007; Robinson et al. 2016) and the risk of developing postoperative AF (Jacob et al. 2015; van Osch et al. 2015). Also, dexametha- 
sone had been proved to inhibit cardiac injury by inducing the transcriptional activation of the anti-apoptotic $\mathrm{Bcl}-\mathrm{xL}$ gene (Xu et al. 2011). However, dexamethasone may be only suitable for some specific cases because the incidence of AF in patients undergoing combined coronary artery bypass graft and valve surgery was not affected by dexamethasone (Yared et al. 2007). Artemisinin was demonstrated to exert protective effects against doxorubicin-induced cardiotoxic-
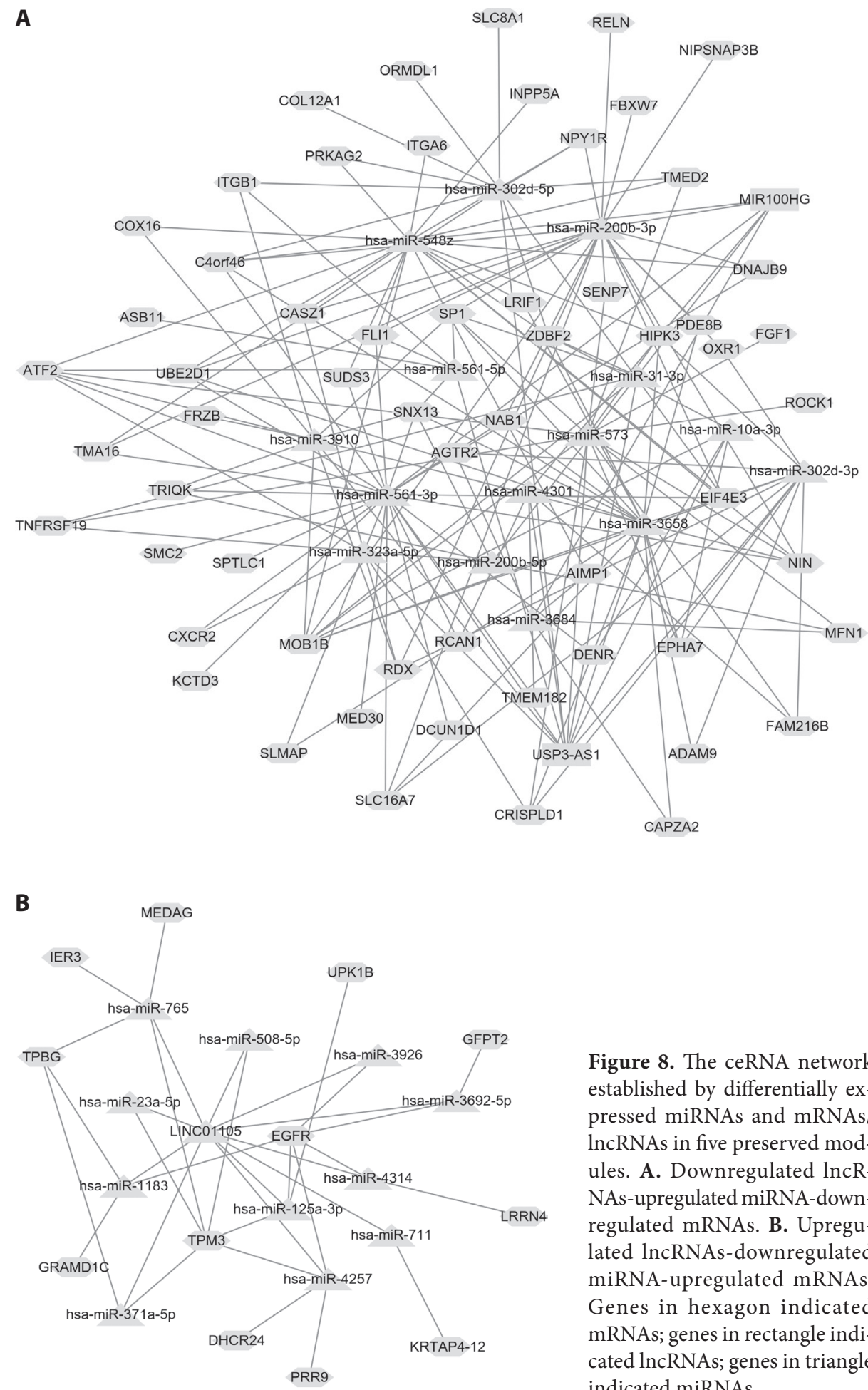

Figure 8. The ceRNA network established by differentially expressed miRNAs and mRNAs/ lncRNAs in five preserved modules. A. Downregulated lncRNAs-upregulated miRNA-downregulated mRNAs. B. Upregulated lncRNAs-downregulated miRNA-upregulated mRNAs. Genes in hexagon indicated mRNAs; genes in rectangle indicated lncRNAs; genes in triangle indicated miRNAs. 
ity or myocardial ischemia-reperfusion injury by reducing the expression of pro-apoptotic caspase-3, caspase- 1 and deactivation of the NLRP3 and NF-kappaB-mediated inflammatory pathways (Gu et al. 2012; Aktaş et al. 2020; Wang et al. 2020). Grisanti et al. (2014) found $\beta$-Adrenergic receptor-mediated transactivation of EGFR could decrease the apoptosis of cardiomyocytes via activation of extracellular-signal-regulated kinase (ERK)-1/2 and Akt pathway and then downregulation of pro-apoptotic caspase-3 activity. The use of EGFR inhibitor AG1478 (Chen et al. 2012) or EGFR siRNA (Chen et al. 2012; Miao et al. 2015) attenuated ERK activation and partially reduced the survival of cardiomyocytes. Hereby, LINC01105 may exert similar anti-apoptotic functions with EGFR in AF.

During the ceRNA mechanisms, lncRNAs could interact with miRNAs to regulate target mRNAs. Thus, lncRNAsrelated miRNAs may be crucial targets for AF, such as LINC01105-miR-125a-3p-EGFR, MIR100HG-miR-200b3p-FBXW7, and MIR100HG-miR-561-3p-CXCR2. Some miRNAs had been revealed to be associated with AF or cardiomyocyte apoptosis, although their interactions with lncRNAs/mRNAs remained unclear. The expression of miR125 a was identified to be dramatically upregulated in patients with late recurrence of AF (Shen et al. 2018) and ischemia/ reperfusion-injured myocardium and $\mathrm{H}_{2} \mathrm{O}_{2}$-induced cardiomyocytes (Yan et al. 2019). Knockdown of miR-125a-5p significantly suppressed $\mathrm{H}_{2} \mathrm{O}_{2}$-induced cardiomyocyte apoptosis (Yan et al. 2019). Overexpression of miR-200b-3p could inhibit cardiomyocyte apoptosis (Xu et al. 2019). In agreement with these studies, we also found miR-125a was highly expressed, while miR-200b was lowly expressed in AF patients compared with SR ones.

\section{Conclusion}

The present study identified two novel lncRNAs (MIR100HG and LINC01105) associated with AF. They may function by co-expressing with (MIR100HG-ROCK1/FBXW7/ UBE2D1, LINC01105-EGFR) mRNAs or sponging miRNAs for mRNAs (LINC01105-miR-125a-3p-EGFR, MIR100HGmiR-200b-3p-FBXW7, MIR100HG-miR-561-3p-CXCR2) to regulate cardiomyocyte apoptosis and atrial fibroblast proliferation, ultimately leading to the development of AF. Dexamethasone may target MIR100HG, while artemisinin may target hub DEGs to reverse the outcomes of AF patients. However, subsequent collection of clinical samples (female and male, respectively) and performance of in vitro and in vivo studies are needed to confirm their expression (especially UBE2D1 which was not studied previously and ROCK1 which was only found to be differentially expressed in male AF samples), association with the development of $\mathrm{AF}$ and regulatory mechanisms.
Funding. This work was supported by National Natural Science Foundation of China Youth Science Foundation Project (No.81700304, 81900314, 82000313), the Scientific Research Fund Project of Key Laboratory of Second Hospital of Tianjin Medical University (No.2019ZDSYS11), the Science \& Technology Development Fund of Tianjin Education Commission for Higher Education (No. 2017KJ205, 2018KJ058, 2020KJ168), Tianjin Natural Science Foundation (No. 17JCYBJC27800).

Availability of data and materials. All microarray data (GSE79768, GSE115574 and GSE68475) were downloaded from GEO database (http://www. ncbi.nlm.nih.gov/geo/).

Conflict of interests. The authors declare that they have no competing interests.

\section{References}

Aktaş I, Özmen Ö, Tutun H, Yalçın A, Türk A (2020): Artemisinin attenuates doxorubicin induced cardiotoxicity and hepatotoxicity in rats. Biotech. Histochem. 95, 121-128

https://doi.org/10.1080/10520295.2019.1647457

Ball J, Thompson DR, Ski CF, Carrington MJ, Gerber T, Stewart S (2015): Estimating the current and future prevalence of atrial fibrillation in the Australian adult population. Med. J. Aust. 202, 32-35 https://doi.org/10.5694/mja14.00238

Cao F, Li Z, Ding WM, Yan L, Zhao QY (2019): LncRNA PVT1 regulates atrial fibrosis via miR-128-3p-SP1-TGF- $\beta 1$-Smad axis in atrial fibrillation. Mol. Med. 25, 7 https://doi.org/10.1186/s10020-019-0074-5

Cao J, Zhang S (2014): A Bayesian extension of the hypergeometric test for functional enrichment analysis. Biometrics 70, 84-94 https://doi.org/10.1111/biom.12122

Chen FY, Zhou ZY, Zhang KJ, Pang J, Wang SM (2020): Long noncoding RNA MIR100HG promotes the migration, invasion and proliferation of triple-negative breast cancer cells by targeting the miR-5590-3p/OTX1 axis. Cancer Cell Int. 20, 508 https://doi.org/10.1186/s12935-020-01580-6

Chen H, Ma N, Xia J, Liu J, Xu Z (2012): ß2-Adrenergic receptorinduced transactivation of epidermal growth factor receptor and platelet-derived growth factor receptor via Src kinase promotes rat cardiomyocyte survival. Cell Biol. Int. 36, 237-244 https://doi.org/10.1042/CBI20110162

Chen HC, Chang JP, Chang TH, Lin YS, Huang YK, Pan KL, Fang CY, Chen CJ, Ho WC, Chen MC (2015): Enhanced expression of ROCK in left atrial myocytes of mitral regurgitation: a potential mechanism of myolysis. BMC Cardiovasc. Disord. 15, 33 https://doi.org/10.1186/s12872-015-0038-9

Chen Q, Feng C, Liu Y, Li QF, Qiu FY, Wang MH, Shen ZD, Fu GS (2019a): Long non-coding RNA PCAT-1 promotes cardiac fibroblast proliferation via upregulating TGF- $\beta 1$. Eur. Rev. Med. Pharmacol. Sci. 23, 10517-10522

Chen Y, Su F, Han J, Jiao P, Guo W (2018): Expression of Rho kinase and its mechanism in the left atrial appendage in patients with atrial fibrillation. Heart Surg. Forum. 21, E044-e048 https://doi.org/10.1532/hsf.1851 
Chen Z, Su X, Shen Y, Jin Y, Luo T, Kim IM, Weintraub NL, Tang Y (2019b): MiR322 mediates cardioprotection against ischemia/ reperfusion injury via FBXW7/notch pathway. J. Mol. Cell Cardiol. 133, 67-74

https://doi.org/10.1016/j.yjmcc.2019.05.020

Dennis G, Sherman BT, Hosack DA, Yang J, Gao W, Lane HC, Lempicki RA (2003): DAVID: Database for Annotation, Visualization, and Integrated Discovery. Genome Biol. 4, P3 https://doi.org/10.1186/gb-2003-4-5-p3

Du J, Li Z, Wang X, Li J, Liu D, Wang X, Wei J, Ma S, Zhang Y, Hou Y (2020): Long noncoding RNA TCONS-00106987 promotes atrial electrical remodelling during atrial fibrillation by sponging miR-26 to regulate KCNJ2. J. Cell. Mol. Med. 24, $12777-12788$ https://doi.org/10.1111/jcmm.15869

Dweep H, Gretz N (2015): miRWalk2.0: a comprehensive atlas of microRNA-target interactions. Nat. Methods 12, 697-697 https://doi.org/10.1038/nmeth.3485

Fan G, Wei J (2020): Identification of potential novel biomarkers and therapeutic targets involved in human atrial fibrillation based on bioinformatics analysis. Kardiol. Pol. 78, 694-702 https://doi.org/10.33963/KP.15339

Grisanti LA, Talarico JA, Carter RL, Yu JE, Repas AA, Radcliffe SW, Tang HA, Makarewich CA, Houser SR, Tilley DG (2014): $\beta$-Adrenergic receptor-mediated transactivation of epidermal growth factor receptor decreases cardiomyocyte apoptosis through differential subcellular activation of ERK1/2 and Akt. J. Mol. Cell Cardiol. 72, 39-51

https://doi.org/10.1016/j.yjmcc.2014.02.009

Gu Y, Wang X, Wang X, Yuan M, Wu G, Hu J, Tang Y, Huang C (2012): Artemisinin attenuates post-infarct myocardial remodeling by down-regulating the NF- $\mathrm{BB}$ pathway. Tohoku J. Exp. Med. 227, 161-170 https://doi.org/10.1620/tjem.227.161

$\mathrm{Hu}$ YF, Chen YJ, Lin YJ, Chen SA (2015): Inflammation and the pathogenesis of atrial fibrillation. Nat. Rev. Cardiol. 12, 230-243 https://doi.org/10.1038/nrcardio.2015.2

Huang Y, Zhang C, Zhou Y (2019): LncRNA MIR100HG promotes cancer cell proliferation, migration and invasion in laryngeal squamous cell carcinoma through the downregulation of miR204-5p. Onco. Targets Ther. 12, 2967-2973 https://doi.org/10.2147/OTT.S202528

Jacob KA, Dieleman JM, Nathoe HM, van Osch D, de Waal EE, Cramer MJ, Kluin J, van Dijk D (2015): The effects of intraoperative dexamethasone on left atrial function and postoperative atrial fibrillation in cardiac surgical patients. Neth. Heart J. 23, 168-173 https://doi.org/10.1007/s12471-014-0638-5

Ke ZP, Xu YJ, Wang ZS, Sun J (2019): RNA sequencing profiling reveals key mRNAs and long noncoding RNAs in atrial fibrillation. J. Cell Biochem. 121, 3752-3763 https://doi.org/10.1002/jcb.29504

Langfelder P, Horvath S (2008): WGCNA: an R package for weighted correlation network analysis. BMC Bioinformatics 9, 559 https://doi.org/10.1186/1471-2105-9-559

Langfelder P, Luo R, Oldham MC, Horvath S, Bourne PE (2011): Is my network module preserved and reproducible? PLoS Comput. Biol. 7, e1001057 https://doi.org/10.1371/journal.pcbi.1001057

Langfelder P, Zhang B, Horvath S (2008): Defining clusters from a hierarchical cluster tree: the Dynamic Tree Cut package for R. Bioinformatics 24, 719-720

https://doi.org/10.1093/bioinformatics/btm563

Li W, Wang L, Wu Y, Yuan Z, Zhou J (2020): Weighted gene coexpression network analysis to identify key modules and hub genes associated with atrial fibrillation. Int. J. Mol. Med. 45, 401-416

https://doi.org/10.3892/ijmm.2019.4416

Liu LJ, Yao FJ, Lu GH, Xu CG, Xu Z, Tang K, Cheng YJ, Gao XR, Wu SH (2016): The Role of the Rho/ROCK Pathway in Ang II and TGF- $\beta 1$-Induced Atrial Remodeling. Plos One 11, e0161625 https://doi.org/10.1371/journal.pone.0161625

Lu J, Xu FQ, Guo JJ, Lin PL, Meng Z, Hu LG, Li J, Li D, Lu XH, An Y (2019): Long noncoding RNA GAS5 attenuates cardiac fibroblast proliferation in atrial fibrillation via repressing ALK5. Eur. Rev. Med. Pharmacol. Sci. 23, 7605-7610

Miao Y, Bi XY, Zhao M, Jiang HK, Liu JJ, Li DL, Yu XJ, Yang YH, Huang N, Zang WJ (2015): Acetylcholine inhibits tumor necrosis factor a activated endoplasmic reticulum apoptotic pathway via EGFR-PI3K signaling in cardiomyocytes. J. Cell Physiol. 230, 767-774 https://doi.org/10.1002/jcp.24800

Morishima M, Iwata E, Nakada C, Tsukamoto Y, Takanari H, Miyamoto S, Moriyama M, Ono K (2016): Atrial fibrillationmediated upregulation of miR-30d regulates myocardial electrical remodeling of the G-protein-gated $\mathrm{K}(+)$ channel, IK.ACh. Circ. J. 80, 1346-1355 https://doi.org/10.1253/circj.CJ-15-1276

Okumura K, Tomita H, Nakai M, Kodani E, Akao M, Suzuki S, Hayashi K, Sawano M, Goya M, Yamashita T, et al. (2020): Risk factors associated with ischemic stroke in Japanese patients with nonvalvular atrial fibrillation. JAMA. Netw. Open 3, e202881 https://doi.org/10.1001/jamanetworkopen.2020.2881

Qian C, Li H, Chang D, Wei B, Wang Y (2019): Identification of functional lncRNAs in atrial fibrillation by integrative analysis of the lncRNA-mRNA network based on competing endogenous RNAs hypothesis. J. Cell Physiol. 234, 11620-11630 https://doi.org/10.1002/jcp.27819

Ritchie ME, Phipson B, Wu D, Hu Y, Law CW, Shi W, Smyth GK (2015): limma powers differential expression analyses for RNAsequencing and microarray studies. Nucleic. Acids Res. 43, e47 https://doi.org/10.1093/nar/gkv007

Robinson E, Kaushal S, Alaboson J, Sharma S, Belagodu A, Watkins C, Walker B, Webster G, McCarthy P, Ho D (2016): Combinatorial release of dexamethasone and amiodarone from a nano-structured parylene- $C$ film to reduce perioperative inflammation and atrial fibrillation. Nanoscale 8, 4267-4275 https://doi.org/10.1039/C5NR07456H

Ruddox V, Sandven I, Munkhaugen J, Skattebu J, Edvardsen T, Otterstad JE (2017): Atrial fibrillation and the risk for myocardial infarction, all-cause mortality and heart failure: A systematic review and meta-analysis. Eur. J. Prev. Cardiol. 24, 1555-1566 https://doi.org/10.1177/2047487317715769

Shen XB, Zhang SH, Li HY, Chi XD, Jiang L, Huang QL, Xu SH (2018): Rs12976445 polymorphism is associated with postablation recurrence of atrial fibrillation by modulating the 
expression of microRNA-125a and Interleukin-6R. Med. Sci. Monit. 24, 6349-6358 https://doi.org/10.12659/MSM.908555

Szklarczyk D, Franceschini A, Wyder S, Forslund K, Heller D, Huerta-Cepas J, Simonovic M, Roth A, Santos A, Tsafou KP (2015): STRING v10: protein-protein interaction networks, integrated over the tree of life. Nucleic Acids Res. 43, D447 https://doi.org/10.1093/nar/gku1003

Tan N, Chung MK, Smith JD, Hsu J, Serre D, Newton DW, Castel L, Soltesz E, Pettersson G, Gillinov AM, et al. (2013): Weighted gene coexpression network analysis of human left atrial tissue identifies gene modules associated with atrial fibrillation. Circ. Cardiovasc. Genet. 6, 362-371

https://doi.org/10.1161/CIRCGENETICS.113.000133

Tang W, Dong K, Li K, Dong R, Zheng S (2016): MEG3, HCN3 and linc01105 influence the proliferation and apoptosis of neuroblastoma cells via the HIF-1 $\alpha$ and p53 pathways. Sci. Rep. 6, 36268 https://doi.org/10.1038/srep36268

Tang Y, Li M, Wang J, Pan Y, Wu FX (2015): CytoNCA: a cytoscape plugin for centrality analysis and evaluation of protein interaction networks. Biosystems 127, 67-72 https://doi.org/10.1016/j.biosystems.2014.11.005

Tsai FC, Lin YC, Chang SH, Chang GJ, Hsu YJ, Lin YM, Lee YS, Wang CL, Yeh YH (2016): Differential left-to-right atria gene expression ratio in human sinus rhythm and atrial fibrillation: Implications for arrhythmogenesis and thrombogenesis. Int. J. Cardiol. 222, 104-112 https://doi.org/10.1016/j.ijcard.2016.07.103

van Osch D, Dieleman JM, van Dijk D, Jacob KA, Kluin J, Doevendans PA, Nathoe HM (2015): Dexamethasone for the prevention of postoperative atrial fibrillation. Int. J. Cardiol. 182, 431-437 https://doi.org/10.1016/j.ijcard.2014.12.094

Wang F, Gao Q, Yang J, Wang C, Cao J, Sun J, Fan Z, Fu L (2020): Artemisinin suppresses myocardial ischemia-reperfusion injury via NLRP3 inflammasome mechanism. Mol. Cell Biochem. 474, 171-180 https://doi.org/10.1007/s11010-020-03842-3

Wang LY, Shen H, Yang Q, Min J, Wang Q, Xi W, Yin L, Le SG, Zhang YF, Xiao J, et al. (2019): LncRNA-LINC00472 contributes to the pathogenesis of atrial fibrillation (Af) by reducing expression of JP2 and RyR2 via miR-24. Biomed. Pharmacother. 120, 109364 https://doi.org/10.1016/j.biopha.2019.109364

Wang X, Fu Q, Song F, Li W, Yin X, Yue W, Yan F, Zhang H, Zhang $\mathrm{H}$, Teng Z, et al. (2018): Prevalence of atrial fibrillation in different socioeconomic regions of China and its association with stroke: Results from a national stroke screening survey. Int. J. Cardiol. 271, 92-97 https://doi.org/10.1016/j.ijcard.2018.05.131

Wu DM, Zhou ZK, Fan SH, Zheng ZH, Wen X, Han XR, Wang S, Wang YJ, Zhang ZF, Shan Q, et al. (2019): Comprehensive RNA-seq data analysis identifies key mRNAs and lncRNAs in atrial fibrillation. Front. Genet. 10, 908 https://doi.org/10.3389/fgene.2019.00908

Wu J, Deng H, Chen Q, Wu Q, Li X, Jiang S, Wang F, Ye F, Ou L, Gao $\mathrm{H}$ (2020a): Comprehensive analysis of differential immunocyte infiltration and potential ceRNA networks involved in the development of atrial fibrillation. Biomed. Res. Int. 2020, 8021208 https://doi.org/10.1155/2020/8021208

Wu N, Li J, Chen X, Xiang Y, Wu L, Li C, Zhang H, Tong S, Zhong L, Li Y (2020b): Identification of long non-coding RNA and circular RNA expression profiles in atrial fibrillation. Heart Lung Circ. 29, e157-e167 https://doi.org/10.1016/j.hlc.2019.10.018

Xu B, Strom J, Chen QM (2011): Dexamethasone induces transcriptional activation of $\mathrm{Bcl}-\mathrm{xL}$ gene and inhibits cardiac injury by myocardial ischemia. Eur. J. Pharmacol. 668, 194-200 https://doi.org/10.1016/j.ejphar.2011.06.019

Xu L, Chen W, Ma M, Chen A, Tang C, Zhang C, Cai L (2019): Microarray profiling analysis identifies the mechanism of miR200b-3p/mRNA-CD36 affecting diabetic cardiomyopathy via peroxisome proliferator activated receptor- $\gamma$ signaling pathway. J. Cell Biochem. 120, 5193-5206 https://doi.org/10.1002/jcb.27795

Yan H, Liang H, Liu L, Chen D, Zhang Q (2019): Long noncoding RNA NEAT1 sponges miR-125a-5p to suppress cardiomyocyte apoptosis via BCL2L12. Mol. Med. Rep. 19, 4468-4474 https://doi.org/10.3892/mmr.2019.10095

Yao L, Zhou B, You L, Hu H, Xie R (2020): LncRNA MIAT/miR133a-3p axis regulates atrial fibrillation and atrial fibrillationinduced myocardial fibrosis. Mol. Biol. Rep. 47, 2605-2617 https://doi.org/10.1007/s11033-020-05347-0

Yared JP, Bakri MH, Erzurum SC, Moravec CS, Laskowski DM, Van Wagoner DR, Mascha E, Thornton J (2007): Effect of dexamethasone on atrial fibrillation after cardiac surgery: prospective, randomized, double-blind, placebo-controlled trial. J. Cardiothorac. Vasc. Anesth. 21, 68-75 https://doi.org/10.1053/j.jvca.2005.10.014

Ye M, Ma J, Liu B, Liu X, Ma D, Dong K (2019): Linc01105 acts as an oncogene in the development of neuroblastoma. Oncol. Rep. 42, 1527-1538 https://doi.org/10.3892/or.2019.7257

Zhang X, Jing W (2018): Upregulation of miR-122 is associated with cardiomyocyte apoptosis in atrial fibrillation. Mol. Med. Rep. 18, 1745-1751 https://doi.org/10.3892/mmr.2018.9124

Zhang YL, Cao HJ, Han X, Teng F, Chen C, Yang J, Yan X, Li PB, Liu Y, Xia YL, et al. (2020a): Chemokine receptor CXCR-2 initiates atrial fibrillation by triggering monocyte mobilization in mice. Hypertension 76, 381-392 https://doi.org/10.1161/HYPERTENSIONAHA.120.14698

Zhang YL, Teng F, Han X, Li PB, Yan X, Guo SB, Li HH (2020b): Selective blocking of CXCR2 prevents and reverses atrial fibrillation in spontaneously hypertensive rats. J. Cell Mol. Med. 24, 11272-11282

https://doi.org/10.1111/jcmm.15694

Zou R, Zhang D, Lv L, Shi W, Song Z, Yi B, Lai B, Chen Q, Yang S, Hua P (2019): Bioinformatic gene analysis for potential biomarkers and therapeutic targets of atrial fibrillation-related stroke. J. Transl. Med. 17, 45 https://doi.org/10.1186/s12967-019-1790-x

Received: February 8, 2021

Final version accepted: April 22, 2021 